\title{
QUASI-PROJECTIVE AND RELATIVE COHOMOLOGICAL DIMENSION OF GROUPS
}

\author{
JUAN M. ALONSO
}

\begin{abstract}
We give a geometric interpretation of the quasi-projective dimension of groups, a notion introduced by Howie and Schneebeli [H-S1] as a generalization of the Identity Property.
\end{abstract}

\section{INTRODUCTION}

Groups of finite quasi-projective dimension (qpd) were introduced by Howie and Schneebeli in [H-S 1] as a generalization of groups having the Identity Property. We will write qpd $G \ll n$ if the trivial $G$-module $\mathbb{Z}$ has a quasiprojective resolution of length $n(n>0)$, i.e. a resolution of the form

$$
0 \rightarrow S \oplus P \rightarrow P_{n-1} \rightarrow \cdots \rightarrow P_{0} \rightarrow \mathbb{Z} \rightarrow 0,
$$

with $P, P_{n-1}, \ldots, P_{0}$ projective $G$-modules, and $S=\bigoplus_{I} \mathbb{Z} G / G_{i}$ for some fixed set $\left(G_{i}\right)_{I}$ of subgroups of $G$. The smallest such $n$ is called the quasiprojective dimension of $G$, written qpd $G$. If there is no $n$ for which qpd $G \ll$ $n$ we set qpd $G=\infty$.

Howie and Schneebeli use only algebraic methods in [H-S 1], and they raise the question of finding a "suitable geometric interpretation of the property of having finite qpd". In this paper we answer this question by identifying the qpd of a group $G$ with the (geometric) dimension of a $\mathrm{CW}$-complex having a $G$-action of a certain type. Recall first the following facts established in [H-S 1]. If qpd $G \ll n$, then:

QP 1. For all $k>n$ the groups $H^{k}(G,-) \cong \prod_{I} H^{k}\left(G_{i},-\right)$ are isomorphic for all coefficient modules.

QP 2. $\left(G_{i}\right)_{I}$ is a complete set of representatives of the conjugacy classes of maximal finite subgroups of $G$.

QP 3. For all $i$ in $I, G_{i}$ is a finite periodic group with period dividing $n$.

It is relevant to this problem to consider the following "natural question" [B, Remark on p. 294] which the author learned to ask from Ken Brown: is the isomorphism in QP 1 an epimorphism for $k=n$ (and, which is the map

Received by the editors July 15, 1988 and, in revised form, April 19, 1989.

1980 Mathematics Subject Classification (1985 Revision). Primary 20J05. 
inducing the isomorphism)? In $\S 2$ we prove

Theorem 1. Let $G$ be a group with qpd $G \ll n$, and let $\left(G_{i}\right)_{I}$ be as in QP 2 . Then

$$
\text { res: } H^{k}(G,-) \rightarrow \prod_{I} H^{k}\left(G_{i},-\right)
$$

is an isomorphism for $k>n$ and an epimorphism for $k=n$, for all coefficient modules.

In $\S 3$ we isolate this property of the restriction map and study it in some detail. A group pair (or pair of groups) $\left(G,\left(G_{i}\right)_{I}\right)$ consists of an arbitrary group $G$ and an arbitrary family $\left(G_{i}\right)_{I}$ of subgroups of $G$. A group pair $\left(G,\left(G_{i}\right)_{I}\right)$ is said to have cohomological dimension $\leq n$, written $\operatorname{cd}\left(G,\left(G_{i}\right)_{I}\right) \leq n$, if res: $H^{k}(G,-) \rightarrow \prod_{I} H^{k}\left(G_{i},-\right)$ is an epimorphism for $k=n$ and an isomorphism for $k>n$, for all coefficient modules. One can show (cf. Corollary 3 below) that if $\operatorname{cd}\left(G,\left(G_{i}\right)_{I}\right) \leq n$ and every $G_{i}$ is a finite group, then the family of $\left(G_{i}\right)_{I}$ is as in QP 2. In this case we say that the relative dimension of $G$, $\operatorname{rd} G$, is $\leq n$. The relationship between qpd and $\mathrm{rd}$ is made precise by

Theorem 2. Given a group $G$ and a positive integer $n$, the following conditions are equivalent:

(i) qpd $G \ll n$,

(ii) $\mathrm{rd} G \leq n$ and each maximal finite subgroup of $G$ is periodic with period dividing $n$.

Our answer to Howie and Schneebeli's question is based on the following topological interpretation of the cohomological dimension of pairs. A cellular action which is free in positive dimensions will be called 0 -unfree. In $\S 4$ we prove the following (for $n>1$; the case where $n=1$ is dealt with in $\S 5$ ):

Theorem 3. Suppose a positive integer $n$ and a group pair $\left(G,\left(G_{i}\right)_{I}\right)$ are given. Then the following conditions are equivalent:

(i) $\operatorname{cd}\left(G,\left(G_{i}\right)_{I}\right) \leq n$,

(ii) there exists an $n$-dimensional, acyclic and 0-unfree $G$-complex $X$ with associated pair $\left(G,\left(G_{i}\right)_{I}\right)$.

When $n=1$ this result is a relative version of the well-known theorem of Stallings and Swan that groups of cohomological dimension one are free, and was proved (for the case where $I=\{i\}$ ) in successive stages (cf. [W1], [Du], [Di], and $\S 5$ below). A consequences of Theorem 3 is that question G6 in Wall's list [W2, p. 392] as to whether $\operatorname{vcd} G=n$ implies the existence of a proper, acyclic $n$-dimensional $G$-complex, has a positive answer for groups of finite rd.

The desired result for groups of finite qpd is

Theorem 4. Given a group $G$ and a positive integer $k$, the following conditions are equivalent:

(i) qpd $G \ll 2 k$, 
(ii) there exists a $2 k$-dimensional acyclic complex $X$ with a 0 -unfree $2 k$ periodic G-action.

Here a proper $G$-action is called $n$-periodic $(n>0)$ if for each 0 -cell $x$ whose isotropy group is nontrivial, we have $H_{*+1}(X, X-x ; \mathbb{Z})=\widetilde{H}_{*}\left(S^{n-1} ; \mathbb{Z}\right)$. It is a consequence of Theorem 2 and well-known results on periodic groups, that when $n=2 k+1$ is odd, qpd $G \ll n$ implies that $\operatorname{cd} G \leq n$. Thus in this case the topological interpretation is given, as is well-known, by Eilenberg-Ganea's Theorem (cf. also Proposition 7 below). When $k>1$, the complex $X$ in Theorem 4 can be taken to be contractible. Theorem 5 below shows that when $k=1, X$ may be taken to be contractible if and only if the group $G$ has the Identity Property.

Finally $\S 5$ deals with the case where $n=1$ in Theorem 3 . We use a trick of Wall [W 1] to deduce the result for arbitrary $I$ from the case where $I$ contains just one element.

Howie and Schneebeli have given in [H-S 2] a topological condition which is sufficient to imply a group has finite qpd. We now briefly discuss this condition and its relation to our characterization. A cellular $G$-action on a $C W$-complex $X$ is called $n$-free [H-S 2] if it restricts to a free action on the $n$-skeleton of $X$. Theorem 1 in [H-S 2] states (when $R=\mathbb{Z}$ ) that if $G$ acts $(n-1)$ freely on a acyclic $n$-dimensional complex, then qpd $G \ll n$ or qpd $G \ll n+$ 1 , depending on whether or not $G$ acts without inversions. Observe that $n$ free actions are not admissible (in the sense of [B]) unless, of course, they are actually free actions. The usual trick to make an action admissible is to subdivide. When applied to an $(n-1)$-free action on a space, this gives a 0 -unfree action on the same space (cf. Theorem 5 below). This process can be reversed in favourable circumstances. For instance, when the space is a triangulated $n$-manifold without boundary, by considering the induced action on the dual triangulation.

This paper is based on my Ph. D. thesis at the University of Stockholm. It is a pleasure to thank Professor Kenneth S. Brown for his aid during the preparation of my thesis. I am also deeply grateful to the members of the departments of mathematics at Cornell University and at the University of Stockholm (specially Professor Jan-Erik Roos). As a member of a badly persecuted generation in Argentina, I would have never been able to complete my thesis without their crucial help at decisive instances.

\section{GROUPS OF FINITE QPD AND THE RESTRICTION MAP}

1. Recall the following useful description of the restriction map [Sw2, Lemma 4.1]:

Let $H$ be a subgroup of a group $G$. Then there are natural isomorphisms $H^{n}(G, M) \cong \operatorname{Ext}_{G}^{n}(\mathbb{Z}, M)$ and $H^{n}(H, M) \cong \operatorname{Ext}_{G}^{n}(\mathbb{Z} G / H, M)$ where $G / H$ is the space of left cosets. Under these isomorphisms, the restriction map 
res: $H^{n}(G, M) \rightarrow H^{n}(H, M)$ corresponds to the map

$$
\operatorname{Ext}_{G}^{n}(\mathbb{Z}, M) \rightarrow \operatorname{Ext}_{G}^{n}(\mathbb{Z} G / H, M)
$$

induced by the map $\varepsilon: \mathbb{Z} G / H \rightarrow \mathbb{Z}$ sending all elements of $G / H$ to 1 .

If we have a pair $\left(G,\left(G_{i}\right)_{I}\right)$ there is a map $H^{k}(G,-) \rightarrow \prod_{I} H^{k}\left(G_{i},-\right)$ whose components are the above restriction maps. It will be called restriction, and denoted res. Set $S=\bigoplus_{I} \mathbb{Z} G / G_{i}$, and let $\varepsilon: S \rightarrow \mathbb{Z}$ be the map whose restriction to each $\mathbb{Z} G / G_{i}$ maps each element of $G / G_{i}$ to 1 . The lemma above implies the existence of natural isomorphisms $\simeq$ such that the diagram below commutes

$$
\begin{array}{cc}
H^{k}(G,-) \stackrel{\text { res }}{\longrightarrow} \prod_{I} H^{k}\left(G_{i},-\right) \\
\downarrow \simeq \\
\downarrow \\
\operatorname{Ext}_{G}^{k}(\mathbb{Z},-) \stackrel{\varepsilon^{*}}{\longrightarrow} \operatorname{Ext}_{G}^{k}(S,-) .
\end{array}
$$

Our aim in this section is to show that if qpd $G \ll n$ and $\left(G_{i}\right)_{I}$ is a complete set of representatives of the conjugacy classes of maximal finite subgroups of $G$, then res is an epimorphism for $k=n$ and an isomorphism for $k>n$, for all $\mathbb{Z} G$-coefficient modules. We begin by constructing a resolution of $\mathbb{Z}$ that contains the available information:

Lemma 1. If qpd $G \ll n$ then $\mathbb{Z}$ has a $\mathbb{Z} G$-projective resolution of the form

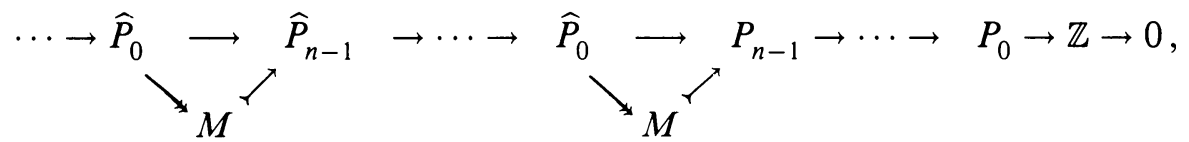

where $M=S \oplus P$ is the kernel of some quasi-projective resolution of length $n$ of $\mathbb{Z}$.

Proof. Choose a quasi-projective resolution $(*) \quad 0 \rightarrow S \oplus P \rightarrow P_{n-1} \rightarrow \cdots \rightarrow$ $P_{0} \rightarrow \mathbb{Z} \rightarrow 0$. We show first that $S$ has a periodic resolution. By QP 3 we have, for each $i \in I$, a resolution

$$
0 \rightarrow \mathbb{Z} \rightarrow \bar{P}_{n-1}^{i} \rightarrow \cdots \rightarrow \bar{P}_{0}^{i} \rightarrow \mathbb{Z} \rightarrow 0
$$

where the $\bar{P}_{j}^{i}$ are $\mathbb{Z} G_{i}$-projective modules for $j=0, \ldots, n-1$ [Sw1]. The desired resolution $0 \rightarrow S \rightarrow \widehat{P}_{n-1}^{\prime} \rightarrow \widehat{P}_{n-2} \rightarrow \cdots \rightarrow \widehat{P}_{1} \rightarrow \widehat{P}_{0}^{\prime} \rightarrow S \rightarrow 0$ is obtained by first applying the exact functor $\mathbb{Z} G \otimes_{G_{i}}$ - to get $0 \rightarrow \mathbb{Z} G / G_{i} \rightarrow$ $\mathbb{Z} G \otimes_{G_{i}} \bar{P}_{n-1}^{i} \rightarrow \cdots \rightarrow \mathbb{Z} G / G_{i} \rightarrow 0$, and then taking the direct sum of these over all $i \in I$. If we now add $P \stackrel{\text { id }}{\longrightarrow} P$ to each end of the resolution just constructed, we obtain a periodic resolution of $M$

$$
0 \rightarrow M \rightarrow \widehat{P}_{n-1} \rightarrow \widehat{P}_{n-2} \rightarrow \cdots \rightarrow \widehat{P}_{0} \rightarrow M \rightarrow 0,
$$

where, e.g., $\widehat{P}_{0}=\widehat{P}_{0}^{\prime} \oplus P$. Finally, the sequence in the lemma is obtained by splicing an infinite number of copies of this last resolution to $(*)$. 
Remark. The sequence in Lemma 1 shows that the group $G$ has period $n$ after $n$-steps [T].

2. The first step is to show that there is some map satisfying the condition:

Proposition 1. There exists a $\mathbb{Z} G$-homomorphism $f: S \rightarrow \mathbb{Z}$ inducing a map

$$
f^{*}: \operatorname{Ext}_{G}^{k}(\mathbb{Z},-) \rightarrow \operatorname{Ext}_{G}^{k}(S,-),
$$

which is an isomorphism for $k>n$ and an epimorphism for $k=n$, for all coefficient modules.

Proof. Arrange two copies of the sequence of Lemma 1 as follows:

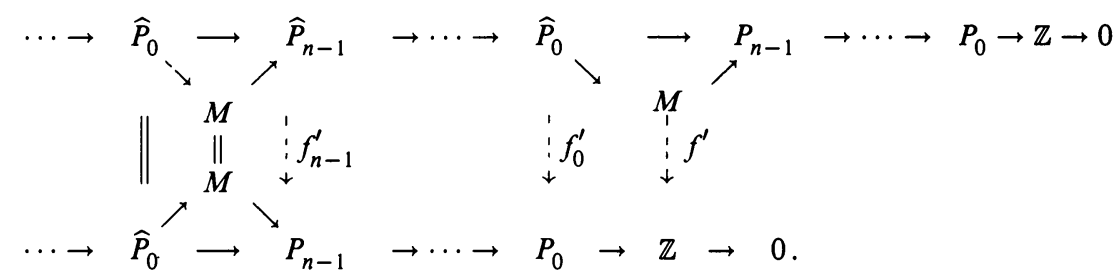

The identity of $M$ lifts to the identity, and we claim that it can also be extended "to the right". In fact, the existence of the maps $f_{n-1}^{\prime}, \ldots, f_{0}^{\prime}, f^{\prime}$ follows from $\operatorname{Ext}_{G}^{k}(\mathbb{Z}, Q)=0$ for $k>n$ and all $G$-projective modules $Q$. And indeed, by QP 1 and 2, we have that $\operatorname{Ext}_{G}^{k}(\mathbb{Z}, Q) \simeq \prod_{I} H^{k}\left(G_{i}, Q\right)=0$, since the groups $G_{i}$ are finite. It is clear that the induced map $\left(f^{\prime}\right)^{*}: \operatorname{Ext}_{G}^{k}(\mathbb{Z},-) \rightarrow \operatorname{Ext}_{G}^{k}(M,-)$ satisfies the conclusion of the Proposition.

Finally we modify $f_{0}^{\prime}$ and $f^{\prime}$ to obtain the map $f: S \rightarrow \mathbb{Z}$ in the statement. We replace the segment $\widehat{P}_{1} \rightarrow \widehat{P}_{0} \oplus P \rightarrow M$ (in dimensions $n+1$ and $n$ of the top row of (1)) by the segment $\widehat{P}_{1} \rightarrow \widehat{P}_{0}^{\prime} \rightarrow S$ (with the notation of Lemma 1). This operation leaves $\operatorname{Ext}_{G}^{k}(\mathbb{Z},-)$ unaltered for $k>n$, so that the argument above produces maps:

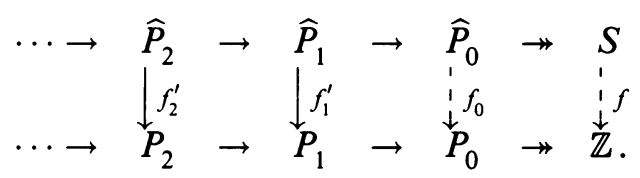

In conclusion, $f$ lifts to a chain map between the resolution of $S$ and $\mathbb{Z}$, which is the identity from dimension $n$ on. This establishes the Proposition.

The next step is to compare the map $f$ just obtained with the restriction map $\varepsilon$. Let $f_{i}$ denote the restriction of $f$ to the direct summand $\mathbb{Z} G / G_{i}$. Since $f_{i}$ is a $G$-homomorphism and $\mathbb{Z}$ has the trivial $G$-structure, we see that there is an integer $m_{i}$ such that $f_{i}\left(g G_{i}\right)=m_{i}$ for all cosets $g G_{i}$ in $G / G_{i}$. Define $t_{i}: \mathbb{Z} G / G_{i} \rightarrow \mathbb{Z} G / G_{i}$ by $t_{i}\left(g G_{i}\right)=m_{i} g G_{i}$ and notice that, identifying $\mathbb{Z} G / G_{i}$ with $\mathbb{Z} G \otimes_{G_{i}} \mathbb{Z}, t_{i}$ corresponds to $\mathrm{id}_{\mathbb{Z} G} \otimes\left(m_{i} \cdot\right)$, where $\left(m_{i} \cdot\right): \mathbb{Z} \rightarrow \mathbb{Z}$ is multiplication by $m_{i}$. Finally, setting $t=\bigoplus_{I} t_{i}: S \rightarrow S$, we have $\varepsilon t=f$. 
Thus the diagram

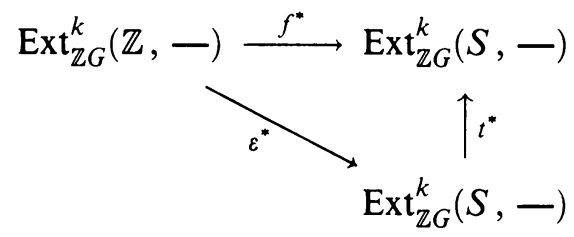

commutes, and Proposition 1 shows that $t^{*}$ is an epimorphism for $k \geq n$ and for all coefficient modules. We now have

Lemma 2. If a map $t: S \rightarrow S$ defined as above induces an epimorphism $t^{*}$ for all $k \geq n$ (and all coefficient modules), then $t^{*}$ is an isomorphism for all $k \geq n$ (and all coefficient modules).

Proof. Since the diagram

$$
\begin{aligned}
& \operatorname{Ext}_{G}^{k}(S,-) \stackrel{t^{*}}{\longrightarrow} \operatorname{Ext}_{G}^{k}(S,-) \\
& \text { l| } \mid \\
& \prod_{I} \operatorname{Ext}_{G}^{k}\left(\mathbb{Z} G / G_{i},-\right) \stackrel{\prod^{\left(t_{i}\right)^{*}}}{\longrightarrow} \prod_{I} \operatorname{Ext}_{G}^{k}\left(\mathbb{Z} G / G_{i},-\right)
\end{aligned}
$$

commutes, it suffices to show that each $\left(t_{i}\right)^{*}$ is an isomorphism for $k \geq n$. Moreover, the following diagram is also commutative

$$
\operatorname{Ext}_{G}^{k}\left(\mathbb{Z} G \otimes_{G_{i}} \mathbb{Z}, N\right) \stackrel{\left(t_{i}\right)^{*}}{\longrightarrow} \operatorname{Ext}_{G}^{k}\left(\mathbb{Z} G \otimes_{G_{i}} \mathbb{Z}, N\right)
$$

$$
\begin{array}{cc}
\| & \text { ॥| } \\
\operatorname{Ext}_{G_{i}}^{k}(\mathbb{Z}, N) \stackrel{\left(m_{i} \cdot\right)^{*}}{\longrightarrow} & \operatorname{Ext}_{G_{i}}^{k}(\mathbb{Z}, N),
\end{array}
$$

where the vertical arrows are the natural change-of-ring isomorphisms, and $N$ is an arbitrary $G$-module. Hence the lemma will follow from the following statement:

$$
\left(m_{i} \cdot\right)^{*} \text { in (2) is an isomorphism for all } k \geq n \text { and all } G_{i} \text {-modules. }
$$

To prove this, note first that $\left(m_{i} \cdot\right)^{*}: H^{k}\left(G_{i}, N\right) \rightarrow H^{k}\left(G_{i}, N\right)$ is multiplication by $m_{i}$ ( $N$ denotes an arbitrary $G_{i}$-module). Now, letting $N=\mathbb{Z}$ and $k=n$ in $(2)$, we see that $\left(m_{i} \cdot\right)^{*}: H^{k}\left(G_{i}, \mathbb{Z}\right) \rightarrow H^{n}\left(G_{i}, \mathbb{Z}\right)$ is an epimorphism. By periodicity, $H^{n}\left(G_{i}, \mathbb{Z}\right) \simeq \widetilde{H}^{0}(G, \mathbb{Z})=\mathbb{Z} /\left|G_{i}\right| \mathbb{Z}$, and we conclude that $m$ and $\left|G_{i}\right|$ are relatively prime.

We now prove the statement in the finitely generated case; the general case will follow by a limit argument. Let $N$ be a finitely generated $G_{i}$-module. Then $H^{k}\left(G_{i}, N\right)$ is actually a finite group, because it is torsion (it has exponent dividing $\left|G_{i}\right|$ ) and it is a finitely generated abelian group ( $\mathbb{Z}$ has a $G_{i}$-projective resolution which is finitely generated in each dimension, and $N$ is finitely generated over $\mathbb{Z})$. On the other hand, we can write $H^{k}\left(G_{i}, N\right)=\bigoplus_{p} H^{k}\left(G_{i}, N\right)_{(p)}$ 
for all $k>0$, where $p$ ranges over all the primes dividing the order of $G_{i}$, and $H^{k}\left(G_{i}, N\right)_{(p)}$ is the $p$-primary component of $H^{k}\left(G_{i}, N\right)$. Since $m_{i}$ and $\left|G_{i}\right|$ are relatively prime, we see that $\left(m_{i} \cdot\right)^{*}$ is injective in each primary component, and hence in all of $H^{k}\left(G_{i}, N\right)$. Thus $\left(m_{i} \cdot\right)^{*}$, being an injection of a finite set to itself, is a bijection.

Now let $N$ be an arbitrary $G_{i}$-module. Then $N=\underset{\lim }{\longrightarrow} N_{\lambda}$, where $\left(N_{\lambda}\right)_{\Lambda}$ is the family of all the finitely generated submodules of $\vec{N}$, ordered by inclusion. The natural maps $N_{\lambda} \rightarrow \lim N_{\lambda}$ induce a compatible family of maps $\operatorname{Ext}_{G_{i}}^{k}\left(\mathbb{Z}, N_{\lambda}\right) \rightarrow \operatorname{Ext}_{G_{i}}^{k}\left(\mathbb{Z}, \underline{\lim } N_{\lambda}\right)$ for each $k \geq 0$, and hence a map

$$
\ell: \underset{\lim }{\longrightarrow} \operatorname{Ext}_{G_{i}}^{k}\left(\mathbb{Z}, N_{\lambda}\right) \rightarrow \operatorname{Ext}_{G_{i}}^{k}\left(\mathbb{Z}, \stackrel{\lim }{\longrightarrow} N_{\lambda}\right),
$$

which is an isomorphism for all $k \geq 0$ because $G_{i}$ is finite [B, p. 195]. If we now let $\left(m_{i}^{\lambda} \cdot\right)^{*}: \operatorname{Ext}_{G_{i}}^{k}\left(\mathbb{Z}, N_{\lambda}\right) \rightarrow \operatorname{Ext}_{G_{i}}^{k}\left(\mathbb{Z}, N_{\lambda}\right)$ denote the homomorphism induced by $\left(m_{i}^{\lambda}\right): \mathbb{Z} \rightarrow \mathbb{Z}$, we have the following commutative diagram:

$$
\begin{aligned}
& \stackrel{\lim }{\longrightarrow} \operatorname{Ext}_{G_{i}}^{k}\left(\mathbb{Z}, N_{\lambda}\right) \stackrel{\ell}{\simeq} \operatorname{Ext}_{G_{i}}^{k}(\mathbb{Z}, N) \\
& \simeq \downarrow \downarrow \lim _{\vec{m}\left(m_{i}^{\lambda} \cdot\right)^{*}} \\
& \stackrel{\lim }{\longrightarrow} \operatorname{Ext}_{G_{i}}^{k}\left(\mathbb{Z}, N_{\lambda}\right) \stackrel{\ell}{\simeq} \operatorname{Ext}_{G_{i}}^{k}(\mathbb{Z}, N),
\end{aligned}
$$

where $\underset{\lim }{\longrightarrow}\left(m_{i}^{\lambda}\right)^{*}$ is an isomorphism because each $\left(m_{i}^{\lambda} \cdot\right)^{*}$ is an isomorphism. This establishes the statement and thus the lemma.

Using the lemma of Swan mentioned at the beginning of this section, we can summarize our results so far as follows:

Theorem 1. Let $G$ be a group with apd $G \ll n$, and let $\left(G_{i}\right)_{I}$ be a complete set of representatives of the conjugacy classes of maximal finite subgroups. Then

$$
\text { res: } H^{k}(G,-) \rightarrow \prod_{I} H^{k}\left(G_{i},-\right)
$$

is an isomorphism for $k>n$ and an epimorphism for $k=n$, for all $G$-coefficient modules.

\section{THE RELATIVE DIMENSION OF A GROUP}

1. The property of res established in Theorem 1 is a sort of relative cohomological dimension. This is made precise below.

We assume given an arbitrary group of pair $\left(G,\left(G_{i}\right)_{I}\right)$ and continue to denote the $G$-module $\bigoplus_{I} \mathbb{Z} G / G_{i}$ by $S$ and the kernel of the corresponding map $\varepsilon: S \rightarrow \mathbb{Z}$ by $\Delta$. Recall from [B-E, p. 299] (cf. also [W1, p. 141]) that the pair $\left(G,\left(G_{i}\right)_{I}\right)$ is said to have cohomological dimension $\leq n$ (resp. $=n$ ), written $\operatorname{cd}\left(G,\left(G_{i}\right)_{I} \leq n\right.$ (resp. $\left.=n\right)$, if the projective dimension (over $\mathbb{Z} G$ ) of $\Delta$ is $\leq n-1$ (resp. $=n-1)$. One defines the relative cohomology groups of the pair $\left(G,\left(G_{i}\right)_{I}\right)$ by $H^{k+1}\left(G,\left(G_{i}\right)_{I}, N\right)=\operatorname{Ext}_{G}^{k}(\Delta, N)$ for all $k \geq 0$ 
and all $G$-modules $N$ (relative homology groups are defined analogously, see [B-E]). Applying $\operatorname{Ext}_{G}^{*}(-, N)$ to $0 \rightarrow \Delta \rightarrow S \rightarrow \mathbb{Z} \rightarrow 0$ one obtains the long exact sequence for relative cohomology:

$$
\cdots \rightarrow H^{k}(G,-) \stackrel{\text { res }}{\longrightarrow} \prod_{I} H^{k}\left(G_{i},-\right) \rightarrow H^{k+1}\left(G,\left(G_{i}\right),-\right) \rightarrow \cdots .
$$

We now have

Lemma 3. Given a pair $\left(G,\left(G_{i}\right)_{I}\right)$ and an integer $n>0$, the following conditions are equivalent:

(i) res: $H^{k}(G,-) \rightarrow \prod_{I} H^{k}\left(G_{i},-\right)$ is an epimorphism for $k=n$ and an isomorphism for $k>n$, for all coefficient modules,

(ii) $\operatorname{cd}\left(G,\left(G_{i}\right)_{I}\right) \leq n$,

(iii) there is a resolution of the form $0 \rightarrow P_{n} \rightarrow \cdots P_{1} \rightarrow P_{0} \oplus S \stackrel{\eta}{\longrightarrow} \mathbb{Z} \rightarrow 0$ where $P_{n}, \ldots, P_{0}$ are G-projective and $\eta \mid S=\varepsilon$.

Remark. Condition (iii) above is the algebraic analog of our topological interpretation of the property of a pair of having finite cohomological dimension (see Theorem 3 below).

Proof. the equivalence of (i) and (ii) follows immediately from the exact sequence for relative cohomology. If (ii) holds then $\Delta$ has a projective resolution $0 \rightarrow P_{n} \rightarrow \cdots \rightarrow P_{1} \rightarrow \Delta \rightarrow 0$, and splicing this with $0 \rightarrow \Delta \rightarrow S \rightarrow \mathbb{Z} \rightarrow 0$ we get a resolution as in (iii). To see that (iii) implies (ii), let $\Delta^{\prime}=\operatorname{ker} \eta$ and note that the relation $\eta \mid S=\varepsilon$ yields the commutative diagram

$$
\begin{aligned}
& 0 \rightarrow \underset{j \downarrow}{\Delta} \rightarrow \underset{\downarrow}{S} \stackrel{\varepsilon}{\rightarrow} \underset{\|}{\mathbb{Z}} \rightarrow 0 \\
& 0 \rightarrow \Delta^{\prime} \rightarrow S \oplus P_{0} \stackrel{\eta}{\rightarrow} \mathbb{Z} \rightarrow 0 .
\end{aligned}
$$

It follows that $\operatorname{ker} j=0$ and that $\operatorname{coker} j \cong P_{0}$. Thus $\Delta^{\prime} \cong \Delta \oplus P_{0}$ and $\mathrm{pd}_{G} \Delta=\mathrm{pd}_{G} \Delta^{\prime} \leq n-1$.

The corollaries below give examples of group pairs of finite cd.

Corollary 1. Let $G$ be the fundamental group of a graph of groups with vertex groups $\left(G_{v}\right)_{V}$ and edge groups $\left(G_{e}\right)_{E}$, and let $n>0$ be an integer. Then $\operatorname{cd}\left(G,\left(G_{v}\right)_{V}\right) \leq n$ if and only if $\operatorname{cd} G_{e} \leq n-1$ for all $e \in E$.

Proof. Recall from [C] that if $G$ is as above there is an exact sequence

$$
0 \rightarrow \bigoplus_{E} \mathbb{Z} G / G_{e} \rightarrow \bigoplus_{V} \mathbb{Z} G / G_{v} \stackrel{\varepsilon}{\rightarrow} \mathbb{Z} \rightarrow 0,
$$

so that in this case $\Delta=\bigoplus_{E} \mathbb{Z} G / G_{e}$ and the corollary follows immediately from (ii) in Lemma 3.

Corollary 2. Let $n>0$ and suppose that $\operatorname{cd}(G, H) \leq n$, where $H$ is the fundamental group of a graph of groups with vertex groups $\left(H_{v}\right)_{V}$ and edge groups $\left(H_{e}\right)_{E}$. If for all $e \in E, \operatorname{cd} H_{e} \leq n-1$, then $\operatorname{cd}\left(G,\left(H_{v}\right)_{V}\right) \leq n$. 
Proof. The map res: $H^{k}(G,-) \rightarrow \prod_{V} H^{k}\left(H_{v},-\right)$ is the composite

$$
H^{k}(G,-) \stackrel{\text { res }}{\longrightarrow} H^{k}(H,-) \stackrel{\text { res }}{\longrightarrow} \prod_{V} H^{k}\left(H_{v},-\right) \text {. }
$$

The first res (respectively, the second) is an epimorphism for $k=n$ and an isomorphism for $k>n$ by hypothesis (resp., by Corollary 1 ).

2. In this section we introduce the relative dimension of a group and relate it to its virtual cohomological dimension (vcd). We begin by establishing relative versions of well-known properties of the absolute cohomological dimension. The next lemma is proved by applying the usual proof (see e.g. [B, p. 186]) to $\operatorname{Ext}_{G}^{*}(\Delta,-)$ (instead of applying it to $H^{*}(G,-)=\operatorname{Ext}_{G}^{*}(\mathbb{Z},-)$ ):

Lemma 4. If $\operatorname{cd}\left(G,\left(G_{i}\right)_{I}\right)<\infty$ then

$$
\operatorname{cd}\left(G,\left(G_{i}\right)_{I}\right)=\sup \left\{n \mid \operatorname{Ext}_{G}^{n-1}(\Delta, F) \neq 0 \text { for some free } G \text {-module } F\right\} .
$$

Next, suppose a pair $\left(G,\left(G_{i}\right)_{I}\right)$ and a subgroup $H$ of $G$ are given. Then there is a family of subgroups of $H$ induced by the family $\left(G_{i}\right)_{I}$ defined as follows (we follow [B-E]; similar constructions appear in [W1] and [H-S 1]). For each $i \in I$, choose a set $J_{i}$ of representatives of the double cosets $H g G_{i}$, and consider the family of subgroups of $H$

$$
H^{\left(G_{i}\right)}=\left\{H \cap G_{i}^{g} \mid g \in J_{i}, i \in I\right\},
$$

where $G_{i}^{g}=g G_{i} g^{-1}$. Up to conjugation within $H, H^{\left(G_{i}\right)}$ does not depend on the choice of $J=\left\{J_{i} \mid i \in I\right\}$. We thus obtain an induced pair $\left(H, H^{\left(G_{i}\right)}\right)$ and from it, $H$-modules $S_{H}=\bigoplus_{I}\left(\bigoplus_{J_{i}} \mathbb{Z} H / H \cap G_{i}^{g}\right)$ and $\Delta_{H}=\operatorname{ker}\left\{\varepsilon: S_{H} \rightarrow \mathbb{Z}\right\}$. These are obtained from the corresponding $G$-modules $S$ and $\Delta$ by restriction. More precisely, we have the following lemma [B-E, Lemma 7.4] whose proof is included here for the reader's convenience:

Lemma 5. There are $H$-module isomorphism $S \cong S_{H}$ and $\Delta \cong \Delta_{H}$.

Proof. The permutation module $S=\bigoplus_{I} \mathbb{Z} G / G_{i}$ is the free abelian group on a $G$-set $X$ whose decomposition into $G$-orbits has the form $X=\bigsqcup_{I} G / G_{i}$. The $H$-orbit decomposition of $X$ has the form $\amalg_{I}\left(\amalg_{J_{i}} H / H \cap G_{i}^{g}\right)$ so that, as $H$-module, $S \cong S_{H}$.

More formally, there is a canonical $H$-modules homomorphism $\mu: S \rightarrow S_{H}$ give by $\mu\left(\bar{g} G_{i}\right)=h\left(H \cap G_{i}^{g}\right)$ where $\bar{g}$ in $G$ is arbitrary, and $h \in H, g \in$ $J_{i}, g_{i} \in G_{i}$ are chosen so that $\bar{g}=h g g_{i}$. This map is well-defined and its inverse is given by $\mu^{-1}\left(h\left(H \cap G_{i}^{g}\right)\right)=h g G_{i}$. Evidently $\mu$ commutes with the augmentations and thus induces an isomorphism $\Delta \cong \Delta_{H}$.

Lemma 6. Let $\left(G,\left(G_{i}\right)_{I}\right)$ be a pair of groups and $H$ a subgroup of $G$. Then

(i) $\operatorname{cd}\left(H, H^{\left(G_{i}\right)}\right) \leq \operatorname{cd}\left(G,\left(G_{i}\right)_{I}\right)$,

(ii) if $\operatorname{cd}\left(G,\left(G_{i}\right\rangle_{I}\right)<\infty$ and $H$ has finite index in $G$, then $\operatorname{cd}\left(H, H^{\left(G_{i}\right)}\right)=$ $\operatorname{cd}\left(G,\left(G_{i}\right)_{I}\right)$. 
Proof. If $\operatorname{cd}\left(G,\left(G_{i}\right)_{I}\right)=\infty$ there is nothing to prove. If the cohomological dimension of the pair is finite, then for some $n, \mathbb{Z}$ has a $G$-resolution of the form $0 \rightarrow P_{n} \rightarrow \cdots \rightarrow P_{0} \oplus S \rightarrow \mathbb{Z} \rightarrow 0$ and by Lemma 5 , its restriction to $H$ has the form in (iii) of Lemma 3. This proves (i).

(ii) If $\operatorname{cd}\left(G,\left(G_{i}\right)_{I}\right)=n<\infty$, then by Lemma $4, \operatorname{Ext}_{G}^{n-1}(\Delta, F) \neq 0$ for some free $G$-module $F$. Let $F^{\prime}$ be the free $H$-module of the same rank as $F$. Then $F \cong \mathbb{Z} G \otimes_{\mathbb{Z} H} F^{\prime} \cong \operatorname{Hom}_{H}\left(\mathbb{Z} G, F^{\prime}\right)$, where the second isomorphism is a consequence of the fact that $H$ has finite index in $G$. Then

$$
\begin{aligned}
0 & \neq \operatorname{Ext}_{G}^{n-1}(\Delta, F) \cong \operatorname{Ext}_{G}^{n-1}\left(\Delta, \operatorname{Hom}_{H}\left(\mathbb{Z} G, F^{\prime}\right)\right) \\
& \cong \operatorname{Ext}_{H}^{n-1}\left(\Delta, F^{\prime}\right) \text { by a change-of-ring-isomorphism } \\
& \cong \operatorname{Ext}_{H}^{n-1}\left(\Delta_{H}, F^{\prime}\right) \text { by Lemma } 5 .
\end{aligned}
$$

By Lemma $4, \operatorname{cd}\left(H, H^{\left(G_{i}\right)}\right) \geq n$. In view of (i), $\operatorname{cd}\left(H, H^{\left(G_{i}\right)}\right)=\operatorname{cd}\left(G,\left(G_{i}\right)_{I}\right)$.

When the family $\left(G_{i}\right)_{I}$ in the pair of groups $\left(G,\left(G_{i}\right)_{I}\right)$ consists only of finite groups, $\operatorname{cd}\left(G,\left(G_{i}\right)_{I}\right)$ will be called the relative dimension of $G$, denoted $\operatorname{rd} G$. This omission of the family of subgroups is justified by the fact (see Corollary 3 below) that when such a pair has finite cohomological dimension, the family $\left(G_{i}\right)_{I}$ is a complete set of representatives of the conjugacy classes of the maximal finite subgroups of $G$. Thus, when $G$ is torsion-free, we recover the usual cohomological dimension of $G$. In this case $\operatorname{rd~} G=\operatorname{cd} G$. With this notation we have:

Proposition 2. Let $G$ be a virtually torsion-free group. Then $\operatorname{ved} G \leq \operatorname{rd} G$, and if $\mathrm{rd} G$ is finite, then $\operatorname{vcd} G=\operatorname{rd} G$.

Proof. Let $H$ be a torsion-free subgroup of finite index in $G$. Since $H$ has no nontrivial finite subgroups, $\left(H, H^{\left(G_{i}\right)}\right)=(H,\{1\})$ (here $\left(G_{i}\right)$ is a complete set of representatives of the conjugacy classes of maximal finite subgroups of $G$ ). The Proposition now follows from Lemma 6 applied to this induced pair.

The inequality in the Proposition is often strict. For instance, if $G=\mathrm{SL}_{2}(\mathbb{Z})$ then $\operatorname{vcd} G=1$ while $\operatorname{rd} G$ is infinite (by Corollary 7 below).

3. We end this section by making precise the relation between qpd and $\mathrm{rd}$.

Theorem 2. Given a group $G$ and a positive integer $n$, the following conditions are equivalent:

(i) qpd $G \ll n$.

(ii) $\operatorname{rd} G \leq n$ and each maximal finite subgroup of $G$ is periodic with period dividing $n$.

Proof. (i) $\Rightarrow$ (ii). The two conditions follow, respectively, from Theorem 1 and QP 3.

(ii) $\Rightarrow\left(\right.$ i). Let $\left(G_{i}\right)$ be a complete set of representatives of the conjugacy classes of maximal finite subgroups of $G$. Proceeding as in the proof of Lemma 
1, we obtain a periodic resolution of $S=\bigoplus_{I} \mathbb{Z} G / G_{i}$ of length $n: 0 \rightarrow S \rightarrow$ $P_{n-1} \rightarrow \cdots \rightarrow P_{0} \stackrel{g}{\longrightarrow} S \rightarrow 0$. On the other hand, $\operatorname{rd} G=\operatorname{cd}\left(G,\left(G_{i}\right)_{I}\right) \leq n$ so that $\Delta=\operatorname{ker}\{\varepsilon: S \rightarrow \mathbb{Z}\}$ has a projective resolution $0 \rightarrow Q_{n} \rightarrow \cdots \rightarrow Q_{1} \rightarrow$ $\Delta \rightarrow 0$ of length $n-1$. We now lift the inclusion $j: \Delta \rightarrow S$ to a chain map $\tilde{j}$ between these resolutions. Then the mapping cylinder of $\tilde{j}$ has the form

$$
0 \rightarrow S \oplus Q_{n} \rightarrow P_{n-1} \oplus Q_{n-1} \rightarrow \cdots \rightarrow P_{1} \oplus Q_{1} \rightarrow P_{0},
$$

and extending this sequence by $\varepsilon g: P_{0} \rightarrow \mathbb{Z}$ we obtain a quasi-projective resolution of $\mathbb{Z}$ of length $n$, as desired.

A simple example of a group with $r d \neq$ qpd is provided by the modular group $G=\mathbb{Z} / 2 \mathbb{Z} * \mathbb{Z} / 3 \mathbb{Z}$. Indeed, $\operatorname{rd} G=\operatorname{vcd} G=1$, while $\mathbb{Z} / 2 \mathbb{Z}$ and $\mathbb{Z} / 3 \mathbb{Z}$ are periodic with period two. By the Theorem, qpd $G=2$.

\section{TOPOLOGICAL INTERPRETATION}

1. Recall that a $G$ - $C W$-complex (or simply a $G$-complex) is a $C W$-complex $X$ together with an action of the group $G$ on $X$ which permutes the cells. If $\sigma$ is a cell of $X$, the isotropy group of $\sigma$, denote $G_{\sigma}$, is the subgroup of $G$ consisting of those $g$ satisfying $g \sigma=\sigma$. A $G$-complex $X$ is called free (respectively, proper) if for every cell $\sigma$ of $X$, the isotropy group $G_{\sigma}$ is trivial (respectively, finite). It is technically convenient to work with admissible actions (in the sense of [B]); these have the property that the isotorpy group of each cell fixes it pointwise. Free and " 0 -unfree" actions (see below) are always admissible.

As we will show, relative dimension (and hence qpd) is closely related to the following special type of action: we say that $X$ is a 0 -unfree $G$-complex if each cell of positive dimension has trivial isotropy group. Any 0-unfree $G$ complex defines a pair $\left(G,\left(G_{i}\right)_{I}\right)$ by choosing a set $I$ of representatives of the $G$-orbits of the 0 -cells, and letting $G_{i}$ be the isotropy group of $i \in I$. Any such choice will be said to provide a pair associated to the 0-unfree complex. Notice that any two pairs associated to a given 0 -unfree complex have the same cohomological dimension.

A 0-unfree action of a group on a finite dimensional, acyclic complex imposes severe restrictions to its finite subgroups, as shown by the next proposition. This result is a relative (and topological) version of the fact that a group of finite cohomological dimension (i.e., the case when all the isotropy groups $G_{i}$ are trivial) has no nontrivial finite subgroups. See Proposition $3^{\prime}$ ( $\S 4.3$ below) for a purely algebraic formulation and more comments. The $n$-skeleton of $X$ will be denoted $X^{(n)}$.

Proposition 3. Let $X$ be a finite dimensional, acyclic and 0-unfree G-complex, and let $F$ be a nontrivial finite subgroup of $G$. Then there exists a unique $x \in X^{(0)}$ such that $F \leq G_{x}$.

Proof. By restricting the action we can consider $X$ as an $F$-complex (with 0unfree action, since $F_{\sigma}=F \cap G_{\sigma}$ ). The fixed-point set $X^{F}$ is a subcomplex. 
Assume first that $F$ has prime order $p$. By "Smith theory" (see, e.g. [B, p. 181]) $H^{*}\left(X^{F} ; \mathbb{Z} / p \mathbb{Z}\right) \cong H^{*}(p t ; \mathbb{Z} / p \mathbb{Z})$ which implies that $X^{F}$ contains exactly one 0 -cell. Thus, the proposition is true in this case.

The rest of the proof proceeds just as in [W 1, p. 149]. Assume, inductively, that $|F|$ is not a prime, and let $M$ be a maximal subgroup of $F$ with $1<$ $|M|<|F|$. By induction, there is a unique $x \in X^{(0)}$ such that $M \leq G_{x}$. If for some $g \in F-M, M$ intersects $M^{g}$ nontrivially, then $M^{g} \cap M \leq G_{g x} \cap G_{x}$ and, by induction, $x=g x$. Hence $g \in G_{x}$ and, by maximality, $F \leq G_{x}$. If, on the other hand, $M$ intersects $M^{g}$ trivially for all $g$ in $F-M$, then $F$ is a Frobenius group, and so has a proper normal subgroup. The desired result is obtained by using this normal complement instead of $M$. Uniqueness is clear in both cases: if $F$ is contained in two isotropy groups, choose a subgroup of $F$ of prime order, and use the first part.

2. We begin by recalling the construction of induced $G$-complexes. Suppose a subgroup $H$ of $G$ and an $H$-complex $X$ are given. The $G$-complex induced from the $H$-complex $X$ is the space $Y:=G \times_{H} X$ defined as the quotient of $G \times X$ (where $G$ is given the discrete topology) by the diagonal $H$-action:

$$
h(g, x)=\left(g h^{-1}, h x\right), \quad h \in H, \quad g \in G, \quad x \in X .
$$

$G$ acts on $Y$ by $g_{1}\left[g_{2}, x\right]=\left[g_{1} g_{2}, x\right]$, where $g_{1}, g_{2} \in G, x \in X$ and [ $g, x]$ denotes the class of $(g, x)$ in $Y$. If $f: X \rightarrow X^{\prime}$ is $H$-equivariant (i.e. $f(h x)=h f(x)$ for all $h$ in $H$ and $x$ in $X)$ then there is a $G$-equivariant map

$$
\operatorname{id}_{G} \times_{H} f: G \times_{H} X \rightarrow G \times_{H} X^{\prime}
$$

defined by $\left(\operatorname{id}_{G} \times_{H} f\right)[g, x]=[g, f(x)]$.

$X$ can be identified with a subspace of $Y$ by $x \rightsquigarrow[1, x]$ and then the action of $G$ on $Y$ extends that of $H$ on $X$. Moreover, if $g_{1}, g_{2} \in G$ then $g_{1} X=g_{2} X$ if and only if $g_{1} H=g_{2} H$, showing that $g X$, for $g \in G / H$, is well-defined. Clearly, we have that $Y=\amalg_{g \in G / H} g X$, the disjoint union of the $g X$. The $C W$-complex structure of $X$ carries over to $g X$ making $Y$ a $G$-complex. Moreover, if $f: X \rightarrow X^{\prime}$ is cellular then so is $\operatorname{id}_{G} \times_{H} f$.

The following proposition is a relative version of a well-known theorem of Eilenberg and Ganea (see, e.g. [B, p. 205]):

Proposition 4. Let $\left(G,\left(G_{i}\right)_{I}\right)$ be a pair of groups, and $n>1$ an integer. The following conditions are equivalent:

(i) $\operatorname{cd}\left(G,\left(G_{i}\right)_{I}\right) \leq n$.

(ii) There exists an acyclic, free $G$-complex $Y$ obtained from $X=$ $\amalg_{I} G \times_{G_{i}} X_{i}$ by attaching cells of dimension $\leq n$, where for each $i \in I$, $X_{i}$ is a contractible, free $G_{i}$-complex.

Remark. In [W 1] Wall states this proposition for $n>2$ (in which case $Y$ can be taken to be contractible, not just acyclic) and studies in detail the case $n=1$. Consequently, we prove here only the case $n=2$ (whose plausibilitiy 
was suggested to me by Ken Brown). We show that the usual proof for $n>2$ [B, pp. 205-208] extends to $n=2$ if one is willing (just as in the absolute case) to compromise for acyclicity. Note, finally, that the proposition indeed reduces, when all the $G_{i}$ are trivial, to the above-mentioned theorem of Eilenberg and Ganea.

Proof. (ii) $\Rightarrow(\mathrm{i})$. Let $C_{*}(X)$ denote the cellular chain complex of $X$. Then $C_{*}(X) \cong \bigoplus_{I} \mathbb{Z} G \otimes_{G_{i}} C_{*}\left(X_{i}\right)$ as $G$-modules and, since $C_{*}\left(X_{i}\right)$ is augmented over $\mathbb{Z}$, it follows that $\mathbb{Z} G \otimes_{G_{i}} C_{*}\left(X_{i}\right)$ is augmented over $\mathbb{Z} G / G_{i}$. Thus $C_{*}(X)$ is augmented over $S=\bigoplus_{I} \mathbb{Z} G / G_{i}$, and we have the commutative diagram:

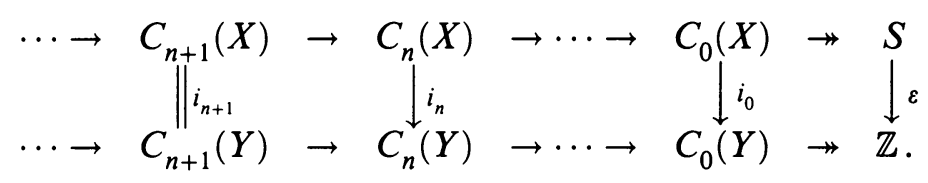

The rows are free resolutions and $\left(i_{k}\right)$, the chain map induced by the inclusion $i: X \rightarrow Y$, is the identity for $k>n$. If we use these resolutions to compute res: $\operatorname{Ext}_{G}^{*}(\mathbb{Z},-) \rightarrow \operatorname{Ext}_{G}^{*}(S,-)$ we see immediately that $\operatorname{cd}\left(G,\left(G_{i}\right)_{I}\right)$ cannot exceed $n$.

(i) $\Rightarrow$ (ii) (for $n=2$ ). For $i \in I$, let $X_{i}$ be a contractible, free $G_{i}$-complex, and define $X$ as in (ii). Choose some 0 -cell of $X$ as the base point *. As above, $C_{*}(X) \rightarrow S$ is a free $G$-resolution of $S$.

By attaching 1-cells connecting the different path-components of $X$, and all their free $G$-translates, we obtain a path-connected, free $G$-complex $X^{\prime}$ containing $X$. It follows that $C_{*}\left(X^{\prime}\right)$ is augmented over $\mathbb{Z}$, and that the inclusion $X \rightarrow X^{\prime}$ induces the chain map:

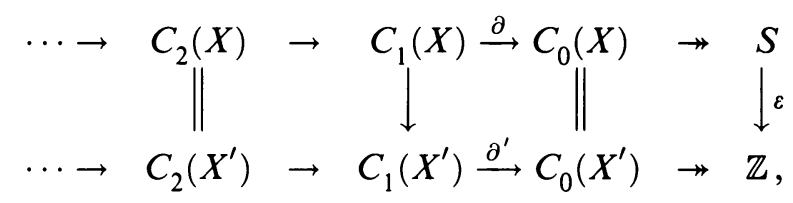

so that $H_{k}\left(X^{\prime}\right)=H_{k}(X)$ for $k>1$, and hence $\widetilde{H}_{k}\left(X^{\prime}\right)=0$ for all $k \neq 1$. Applying the snake lemma to

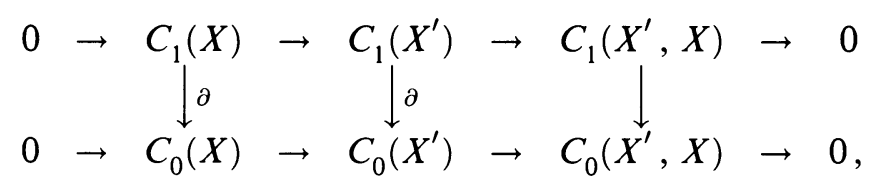

we obtain the exact sequence (note that $\left.C_{0}\left(X^{\prime}, X\right)=0\right)$ :

$$
0 \rightarrow \operatorname{ker} \partial \rightarrow \operatorname{ker} \partial^{\prime} \longrightarrow C_{K} C_{1}\left(X^{\prime}, X\right) \longrightarrow \stackrel{\varepsilon}{\longrightarrow} \mathbb{Z} \rightarrow 0 .
$$

By construction, $C_{1}\left(X^{\prime}, X\right)$ is a free $G$-module and, by hypothesis, $\operatorname{pd}_{G} \Delta \leq 1$. It follows that $K$ is projective and that $\operatorname{ker} \partial^{\prime}=\operatorname{ker} \partial \oplus K$. Note also that $H_{1}\left(X^{\prime}\right) \cong \operatorname{ker} \partial^{\prime} / \operatorname{ker} \partial \cong K$. 
Next, we use Eilenberg's trick (see e.g. [B]) to find a free $G$-module $F$ such that $K \oplus F \cong F$. We then construct a $G$-complex $X^{\prime \prime}$ by choosing a basis $\left\{b_{\beta}\right\}_{B}$ of $F$ over $\mathbb{Z} G$, and attaching the wedge of 1 -spheres $\bigvee_{B} S^{1}$ (and all their free $G$-translates) to $X^{\prime}$ at $*$. Thus $X^{\prime \prime}$ is a free $G$-complex with $C_{1}\left(X^{\prime \prime}\right) \cong$ $C_{1}\left(X^{\prime}\right) \oplus F$ and with $C_{k}\left(X^{\prime \prime}\right) \cong C_{k}\left(X^{\prime}\right)$ for all $k \neq 1$. Moreover, $H_{1}\left(X^{\prime \prime}\right) \cong$ $H_{1}\left(X^{\prime}\right) \oplus F \cong F$, while $\widetilde{H}_{k}\left(X^{\prime \prime}\right)=\widetilde{H}_{k}\left(X^{\prime}\right)$ for all $k \neq 1$.

Since the Hurewicz homomorphism $h: \pi_{1}\left(X^{\prime \prime}, *\right) \rightarrow H_{1}\left(X^{\prime \prime}, *\right)$ is an epimorphism, we can represent the basis elements $b_{\beta}$, i.e. there are maps $f_{\beta}$ : $\left(S^{1}, *\right) \rightarrow\left(X^{\prime \prime}, *\right)$ such that $H_{1}\left(f_{\beta}\right)(l)=b_{\beta}$, where $H_{1}\left(f_{\beta}\right): H_{1}\left(S^{1}, *\right) \rightarrow$ $H_{1}\left(X^{\prime \prime}, *\right)$ and $l \in H_{1}\left(S^{1}, *\right)$ is a generator.

Finally, let $Y$ be the free $G$-complex obtained from $X^{\prime \prime}$ by attaching 2-cells $\left\{e_{\beta}\right\}$ (and all their free $G$-translates) via the maps $f_{\beta}$. The only (possibly) nontrivial portion of the long exact sequence of the pair $\left(Y, X^{\prime \prime}\right)$ is

$$
H_{2} X^{\prime \prime} \rightarrow H_{2} Y \rightarrow H_{2}\left(Y, X^{\prime \prime}\right) \stackrel{\delta}{\longrightarrow} H_{2} X^{\prime \prime} \rightarrow H_{1} Y \rightarrow H_{1}\left(Y, X^{\prime \prime}\right),
$$

where the first and last groups are trivial and, by construction, $\delta$ is an isomorphism (as both $H_{2}\left(Y, X^{\prime \prime}\right.$ ) and $H_{1} X^{\prime \prime}$ are free $G$-modules and $\delta$ establishes a bijection between the respective basis $\left\{e_{\beta}\right\}$ and $\left.\left\{b_{\beta}\right\}\right)$. Thus $\widetilde{H}_{*} Y=0$ so that $Y$ is acyclic and satisfies all the other conditions required in (ii).

Remark. The complex $Y$ above can be taken to be simplicial with simplicial action (i.e. $G$ permutes the simplices). Since $Y$ is constructed inductively, it suffices to show that the construction can be started simplicially and that each successive step can be made simplicially. Now, the $X_{i}$ can be chosen to be simplicial (see e.g. [B, p. 208]). Just as in the case of $C W$-structures, it follows that $G \times{ }_{G} X_{i}$ has a simplicial structure invariant under $G$. So the same is true for $X=\bigsqcup_{I} G \times_{G_{i}} X_{i}$ i.e., the construction can be started simplicially. For the induction step, one uses the simplicial approximation theorem to insure that the attaching maps can be taken to be simplicial (relative to some triangulation of the domain spheres). The space one obtains by attaching cells via such maps is triangulable.

With the notation of Proposition 4, we assume given, for each $i \in I$, and a $G_{i}$-complex $Z_{i}$ and a cellular $G_{i}$-equivariant map $f_{i}: X_{i} \rightarrow Z_{i}$ which is a homotopy equivalence. The following "omnibus" proposition deals with the effect on $Y$ of replacing the subcomplexes $X_{i}$ by the $Z_{i}$.

Proposition 5. Let $\left(G,\left(G_{i}\right)_{I}\right), n, X_{i}, Y, f_{i}$ and $Z_{i}$ be as above. Then there exists a G-complex $Y^{\prime}$ obtained from $Z=\bigsqcup_{I} G \times_{G_{i}} Z_{i}$ by attaching cells of dimension $\leq n$, which is acyclic (resp., contractible) if $Y$ is acyclic (resp., contractible). Moreover, the G-action on $Y^{\prime}$ is 0-unfree (resp., proper, or admissible) if the same is true for each $Z_{i}$.

Proof. Let $X=\bigsqcup_{I} G \times_{G_{i}} X_{i}$ and $Z=\bigsqcup_{I} G \times_{G_{i}} Z_{i}$, and let $f: X \rightarrow Z$ denote the induced map $\bigsqcup_{I}\left(\mathrm{id}_{G} \times_{G} f_{i}\right) . Y^{\prime}$ will then be the space obtained from $Y$ 
by replacing the $X_{i}$ 's by the $Z_{i}$ 's, i.e. define $Y^{\prime}$ to be the push-out of the maps $Z \stackrel{\leftarrow}{\longleftarrow} \stackrel{\text { incl }}{\longrightarrow} Y$. That is, $Y^{\prime}=Y \cup_{f} Z$, a $C W$-complex whose set of $i$-cells is the union of the set of $i$-cells of $Z$ and the set of $i$-cells of $Y-X$.

Since $f$ is $G$-equivariant, $G$ acts on $Y^{\prime}$. Moreover, with the above description of the cells of $Y^{\prime}$, it is clear that $G$ acts cellularly on $Y^{\prime}$, extending the $G$-actions on $Z$ and $Y-X$. Thus the $G$ action is free on $Y-X$ and on $Z$ it is induced from the $G_{i}$-actions on the $Z_{i}$. Hence the isotropy groups of the $G$-action on $Z$ are conjugates (in $G$ ) of the isotropy groups of the $G_{i}$-actions on the $Z_{i}$. All the assertions concerning the $G$-action on $Y^{\prime}$ follow from this.

Now the definition of $Y^{\prime}$ as a push-out yields the following homotopy commutative diagram

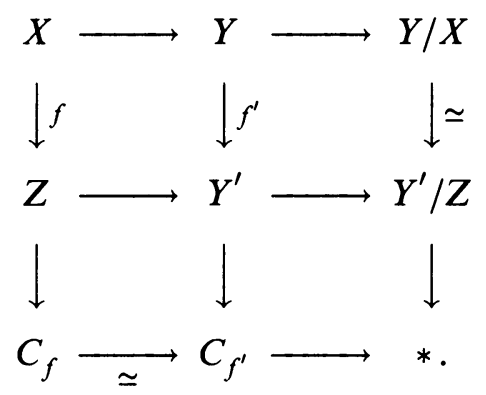

Each row and column in the diagram is a (homotopy) cofibration, and the arrows marked " $\simeq$ " are homotopy equivalences. Since $f$ is a homotopy equivalence, we extract from the diagram the cofibration sequence $Y \stackrel{f^{\prime}}{\longrightarrow} Y^{\prime} \rightarrow *$. It shows that $Y$ and $Y^{\prime}$ have the same homology, so that $Y^{\prime}$ is acyclic if $Y$ is. Applying van Kampen's theorem to the sequence, we see that $\pi_{1} Y^{\prime}$ equals the normal subgroup generated by the image of $\pi_{1} Y$ under $\pi_{1}\left(f^{\prime}\right)$. Thus $Y^{\prime}$ is simply connected if $Y$ is, and it follows that $f^{\prime}$ is actually a homotopy equivalence when $Y$ is contractible.

Lemma 7. Let $X$ and $Y$ be G-complexes and let $K$ be an arbitrary G-subcomplex of $X$. Assume that $X$ is free and $Y$ is contractible. Then any $G$ equivariant map $K \rightarrow Y$ extends to a G-equivariant map $X \rightarrow Y$.

Proof. Extensions exist because $Y$ is contractible, and since the $G$-action is free, it is easy to find an extension which is $G$-equivariant.

There is an obvious way to extend a $G$-action on $X$ to a $G$-action on $C X$, the (unreduced) cone on $X$. Since the isotropy group of the vertex point of the cone is $G$, it follows that $C X$ is 0 -unfree if $X$ is free, and that $C X$ is proper if and only if $G$ is finite. We now have

Lemma 8. Let $X$ be a free, contractible $G$-complex, and $K$ an arbitrary $G$ subcomplex. Then there exists a G-equivariant homotopy equivalence $X \rightarrow C K$ which extends the inclusion $K \rightarrow C K$.

Proof. By Lemma 7, the inclusion $K \rightarrow C K$ extends to a $G$-equivariant map 
$X \rightarrow C K$ which must be a homotopy equivalence since both $X$ and $C K$ are contractible.

3. We now have the following topological interpretation of the cohomological dimension of pairs of groups:

Theorem 3. Suppose given an integer $n \geq 1$, and a group pair $\left(G,\left(G_{i}\right)_{I}\right)$. Then the following conditions are equivalent:

(i) $\operatorname{cd}\left(G,\left(G_{i}\right)_{I}\right) \leq n$.

(ii) There exists an $n$-dimensional, acyclic and 0 -unfree G-complex $X$ with associated pair $\left(G,\left(G_{i}\right)_{I}\right)$.

Remarks. (1) $X$ can be taken to be simplicial with simplicial $G$-action. This follows from the proof below together with the remark after the proof of Proposition 4.

(2) When $n \neq 2, X$ can be taken to be contractible.

(3) When $n=1$ condition (ii) just says that $G$ acts 0 -unfreely on a tree with associated pair $\left(G,\left(G_{i}\right)_{I}\right)$. By Bass-Serre theory, this is further equivalent to the statement that (up to conjugacy) $G=*_{I} G_{i} * F$, where $F$ is a free group (cf. [S, p. 43]). Thus the Theorem in this case is a relative version of the famous theorem of Stallings and Swan that groups of cohomological dimension one are free. It was posed (and solved under extra conditions) by Wall [W 1], later proved by Dunwoody [Du] in the case where $I=\{i\}$ and $G$ is finitely generated over $G_{i}$, and finally proved in general (but still for $I=\{i\}$ ) by Dicks [Di, Theorem 2.11, p. 117]. In $\S 5$ below we show that this last result is valid for arbitrary $I$, thus completing the proof of Theorem 3 for $n=1$.

Proof of Theorem 3 (for $n \geq 2$ ). (i) $\Rightarrow$ (ii). Let $Y$ be the $G$-complex provided by Proposition 4 , and for each $i \in I$, denote by $Z_{i}$ the cone $C X_{i}^{(n-1)}$ on the $(n-1)$-skeleton of $X_{i}$. Apply Lemma 8 to obtain $G$-equivariant maps $f_{i}: X_{i} \rightarrow Z_{i}$ which are homotopy equivalences. The desired complex $X$ is the one provided by Proposition 5 for the above choices of $Z_{i}$ and $f_{i}$. The assertion on the associated pair follows from the description of the isotropy groups of the $G$-action on $X$ given in the proof of Proposition 5.

(ii) $\Rightarrow$ (i) It is a general fact (see e.g. [B, p. 68]) valid for any $G$-complex $X$, that $C_{k}(X)=\bigoplus \mathbb{Z} G \oplus_{G_{\sigma}} \mathbb{Z}_{\sigma}$, where the direct sum is taken over a set $\Sigma_{k}$ of representatives for the $G$-orbits of $k$-cells of $X$, and $\mathbb{Z}_{\sigma}$ is the "orientation module" associated to $\sigma$. Our action is trivially admissible so that we can take $\mathbb{Z}$ (i.e. the integers with trivial $G$-action) instead of $\mathbb{Z}_{\sigma}$. Hence $C_{k}(X)=$ $\bigoplus_{\Sigma_{k}} \mathbb{Z} G / G_{\sigma}$, where $G_{\sigma}$ is trivial if $k>0$, and a conjugate of some $G_{i}$ if $k=0$ (observe that we can take $\Sigma_{0}=I$ ). Thus the cellular complex of $X$ yields the resolution

$$
0 \rightarrow C_{n}(X) \rightarrow \cdots \rightarrow C_{1}(X) \rightarrow C_{0}(X) \stackrel{\varepsilon}{\longrightarrow} \mathbb{Z} \rightarrow 0,
$$

which has the form given in Lemma 3 (iii), since $C_{k}(X)$ is a free $G$-module for $k>0$ and $C_{0}(X)=$ (free $G$-module) $\oplus S$. This completes the proof. 
As an application, the next proposition shows that question G6 of [W 2] has a positive answer for groups of finite relative dimension.

Proposition 6. Let $G$ be a virtually torsion-free group of finite relative dimension. Then there exists a proper, acyclic G-complex of dimension equal to vcd $G$.

Proof. Let rd $G=n<\infty$. By Proposition 2, vcd $G=n$. If $n \geq 1$ the result follows from Theorem 3. When $n=1$ the proposition holds with no assumption on $\mathrm{rd} G$ (see e.g. [B, p. 228]). The result is trivially true when vcd $G=0$.

Theorem 3 also gives the promised reformulation of Proposition 3:

Proposition $3^{\prime}$. Let $\left(G,\left(G_{i}\right)_{I}\right)$ be a pair of finite cohomological dimension and $F$ a nontrivial finite subgroup of $G$. Then there exists a unique $i \in I$ and $a$ unique coset $g G_{i}$ such that $F \leq G_{i}^{g}$.

Remark. This is an unpublished result of Serre (see J. Huebschmann's article [Hu], where an algebraic proof is also given). Wall proved Proposition $3^{\prime}$ in [W 1] under the stronger hypothesis that $\mathrm{cd}\left(G\left(G_{i}\right)_{I}\right)=1$. The same conclusion is obtained in [H-S 1, Theorem 6] under the hypothesis that $G$ has finite qpd.

This proposition has a number of corollaries. Similar ones appear in [H-S 1] for groups of finite qpd, and in [W 1] for pairs of $\mathrm{cd}=1$.

Corollary 3. Suppose that $\operatorname{cd}\left(G,\left(G_{i}\right)_{I}\right)$ is finite and that each $G_{i}$ is a finite group. Then the set $\left(G_{i}\right)_{I}$ is a complete set of representatives of the conjugacy classes of maximal finite subgroups of $G$.

Corollary 4. If $G$ is finite and $\operatorname{cd}\left(G,\left(G_{i}\right)_{I}\right)$ is finite, then some $G=G_{i}$ and the remaining $G_{j}$ are trivial.

Corollary 5. If $\operatorname{cd}\left(G,\left(G_{i}\right)_{I}\right)<\infty$ and $N$ is a finite, nontrivial normal subgroup of $G$, then $G=G_{i}$ for some $i \in I$.

Remark. The other $G_{j}$ need not be trivial, however (but they must be groups of finite cd). For example, take $G=\mathbb{Z} \times \mathbb{Z} / 2 \mathbb{Z}$, with $G_{0}=G$ and $G_{1}=\mathbb{Z}$.

Corollary 6. If $\operatorname{cd}\left(G,\left(G_{i}\right)_{I}\right)$ is finite and $K$ is a nontrivial finite subgroup of some $G_{i}$, then $N_{G}(K)$ (the normalizer of $K$ in $G$ ) is contained in $G_{i}$. In particular, if $\mathrm{rd} G$ is finite and $K$ is as above, then $N_{G}(K)$ must be finite.

Corollary 7. If $\mathrm{rd} G$ is finite and $G$ has nontrivial torsion, then either $G$ is finite or $G$ has trivial center.

Corollary 8. If $\mathrm{rd} G$ is finite and $G$ is abelian and has nontrivial torsion, then $G$ is finite.

4. We now give a topological interpretation of the property of having finite quasi-projective dimension.

Definition. If $X$ is a 0 -unfree, proper $G$-complex, we call the $G$-action $n$ periodic $(n>0)$ if for each 0 -cell $x$ whose isotropy group is nontrivial we have $X_{*+1}(X, X-x ; \mathbb{Z}) \cong \widetilde{H}_{*}\left(S^{n-1} ; \mathbb{Z}\right)$. 
Recall that a $C W$-complex $X$ satisfying $H_{*+1}(X, X-x ; \mathbb{Z}) \cong \widetilde{H}_{*}\left(S^{n-1} ; \mathbb{Z}\right)$ for all $x \in X$ is called a homology manifold of dimension $n$. Any differentiable or topological manifold of dimension $n$ is an example of this. Thus any 0 -unfree, proper action on a homology manifold of dimension $n$ is $n$-periodic. The local homology groups $H_{*}(X, X-x ; \mathbb{Z})$ can be computed, by excision, from any neighbourhood $V$ of $x: H_{*}(X, X-x ; \mathbb{Z}) \cong H_{*}(V, V-x ; \mathbb{Z})$. Finally, note that if $X$ is acyclic then $H_{k}(X, X-x ; \mathbb{Z}) \cong \widetilde{H}_{k-1}^{*}(X-x ; \mathbb{Z})$. We now have

Theorem 4. Given a group $G$ and a positive integer $k$, the following conditions are equivalent:

(i) qpd $G \ll 2 k$,

(ii) There exists a $2 k$-dimensional, acyclic complex $X$ with a 0 -unfree $2 k$ periodic $G$-action.

Proof. $(\Rightarrow)$ Let $\left(G_{i}\right)_{I}$ be a set of representatives of the conjugacy classes of maximal finite subgroups of $G$, and suppose that qpd $G \ll 2 k$. By Theorem $2, \operatorname{rd} G=\operatorname{cd}\left(G,\left(G_{i}\right)_{I}\right) \leq 2 k$ and each $G_{i}$ is periodic with period dividing $2 k$. Assume first that $k>1$. Then by [Sw 1, Proposition 4.1] there exists, for each $i$ in $I$, a $(2 k-1)$-dimensional free $G_{i}$-complex $\bar{X}_{i}$ with the homotopy type of the sphere $S^{2 k-1}$. Note that $\bar{X}_{i}$ can be regarded as the $(2 k-1)$-skeleton of a contractible, free $G_{i}$-complex: simply attach (free $G_{i^{-}}$) cells of dimension $m \geq 2 k$ to kill, inductively, the groups $H_{m-1}\left(\bar{X}^{(m-1)}\right)$. If, on the other hand, $k=1$, then the $G_{i}$ are cyclic groups (since they have period two) and these act freely on the sphere $S^{1}$ which we take as our complex $\bar{X}_{i}$. As above, it can be regarded as the 1 -skeleton of a contractible free $G_{i}$-complex.

Construct now a $G$-complex exactly as in the proof of Theorem 3, using the above $\bar{X}_{i}$ 's as initial data. Such $X$ satisfies all the conditions in (ii) except, possibly, for $2 k$-periodicity. We now check it does. Let $x$ be a vertex of $X$ with nontrivial isotropy group. Then by construction there is $i \in I$ and $g \in G / G_{i}$ such that $x=g v_{i}$, where $v_{i}$ is the vertex of the cone $C \bar{X}_{i}$. Hence $g C \bar{X}_{i}$ is a neighborhood of $g v_{i}$, and we have (all cohomology is with $\mathbb{Z}$ coefficients):

$$
\begin{aligned}
H_{t}(X, X-x) & \cong H_{t}\left(g C \bar{X}_{i}, g C \bar{X}_{i}-g v_{i}\right) \\
& \cong \widetilde{H}_{t-1}\left(g C \bar{X}_{i}-g v_{i}\right) \\
& \cong \widetilde{H}_{t-1}\left(\bar{X}_{i}\right) \cong \widetilde{H}_{t-1}\left(S^{2 k-1}\right),
\end{aligned}
$$

where the map previous to the last is an isomorphism because $g C \bar{X}_{i}-g v_{i}$ retracts to $\bar{X}_{i}$.

$(\Leftarrow)$ Let $X$ be as in (ii) with associated pair $\left(G_{i}\right)_{I}$. Then $\operatorname{rd} G \leq 2 k$ by Theorem 3 and, by Proposition 3 , the family $\left(G_{i}\right)_{I}$ is a complete set of representatives of the conjugacy classes of maximal finite subgroups of $G$. By Theorem 2 , it suffices to show that each $G_{i}$ is periodic with period dividing $2 k$. 
But $G_{i}$ acts freely on $X-i$ (cfr. definition of associated pair) and $\widetilde{H}_{*}(X-i) \cong$ $H_{*+1}(X, X-i) \cong \widetilde{H}_{*}\left(S^{2 k-1}\right)$ because the $G$-action is $2 k$-periodic. This implies that $G_{i}$ has periodic cohomology of period dividing $2 k$ (see e.g. [B, pp. 180181]). The proof is now complete.

Conditions (i) and (ii) in Theorem 4 are no longer equivalent for odd integers $2 k+1$. In fact, (i) is in this case equivalent to cd $G \leq 2 k+1$ (since nontrivial finite groups cannot have odd period) and this is well known to be equivalent to the existence of a free, acyclic $G$-complex of dimension $\leq 2 k+1$. As for (ii), we have the following proposition which should be compared with Theorem 1(b) of [H-S 2]:

Proposition 7. Let $G$ be a group that acts 0 -unfreely and $(2 k+1)$-periodically on an acyclic, $(2 k+1)$-dimensional complex $X$. Then either $\operatorname{cd} G \leq 2 k+1$, or the maximal finite subgroups of $G$ are all isomorphic to $\mathbb{Z} / 2 \mathbb{Z}$ and qpd $G \leq 2 k+2$. Proof. Since the action is proper, if $G$ has no torsion then it acts freely and thus cd $G \leq 2 k+1$. If $G$ has torsion, then the nontrivial isotropy groups must have order 2 (see, e.g. [B, p. 182, Exercise 3]) and they are maximal among the finite subgroups by Proposition 3. Theorem 2 completes the proof.

Remark. I do not know whether the converse is true.

As with Theorem 3, the word "acyclic" in Theorem 4 can be replaced by "contractible" provided that $k \neq 1$. It is not known whether this can also be done in dimension 2: this is the Eilenberg-Ganea problem. The next theorem studies the effect of having a contractible complex of dimension 2 in Theorem 4. Following [H-S 2] we say that a presentation $\langle U \mid R\rangle$ of a group $G$ has the identity property if the following conditions are satisfied:

(i) The relation module is isomorphic to $\bigoplus_{r \in R} C_{r}$, where $C_{r}$ is the cyclic $\mathbb{Z} G$-submodule generated by the image in the relation module of the relator $r$.

(ii) There is an isomorphism $C_{r} \cong \mathbb{Z} G / G_{r}$, where $G_{r}$ is the image in $G$ of the centralizer of $r$ in the free group $F(U)$.

A group $G$ has the identity property if some presentation of $G$ has the identity property. We now have:

Theorem 5. The following conditions on a group $G$ are equivalent:

(i) $G$ has the identity property.

(ii) $G$ acts on a contractible 2-dimensional complex in such a way that the action, when restricted to the 1-skeleton, is free.

(iii) There exists a contractible 2-dimensional complex with a 0-unfree 2periodic G-action.

Proof. The equivalence of (i) and (ii) is a Theorem of Howie and Schneebeli [H-S 2]. The proof that (ii) implies (iii) is by subdividing the 2-cells that have a nontrivial isotropy group. In fact, let $Y$ be a complex as in (ii). We may assume, by subdividing if necessary, that $Y^{(1)}$ is simplicial. Choose a set $\Sigma$ of representatives for the 2-cells with nontrivial isotropy group, modulo $G$. For $\sigma$ 
in $\Sigma$, choose an attaching map $f_{\sigma}^{\prime}: S^{1} \rightarrow Y^{(1)}$, and use simplicial approximation to obtain a simplicial map $f_{\sigma}$ homotopic to $f_{\sigma}^{\prime}$. Delete from $Y$ the 2-cells $D=\left\{g \sigma \mid g \in G / G_{\sigma}, \sigma \in \Sigma\right\}$ and replace them by new 2-cells attached via the maps $g f_{\sigma}$. These 2-cells are then simplicial cones on the closed paths $g f_{\sigma}\left(S^{1}\right)$, with vertices $g v_{\sigma}$. The 2-complex $Y^{\prime}$ thus obtained is homotopically equivalent to $Y$, and hence contractible. Moreover, the free $G$-action on $Y \backslash D$ extends in an obvious way to a $G$-action on $Y^{\prime}$ that is clearly 0 -unfree. We need only check that this action is 2-periodic. Indeed,

$$
H_{*+1}\left(Y^{\prime}, Y^{\prime} \backslash g v_{\sigma}\right) \cong H_{*+1}\left(C\left[g f_{\sigma}\left(S^{1}\right)\right], C\left[g f_{\sigma}\left(S^{1}\right) \backslash g v_{\sigma}\right]\right) \cong \widetilde{H}_{*}\left(S^{1}\right)
$$

(iii) $\Rightarrow$ (ii). Let $Y$ be a 2-complex as in (iii). We assume, as before, that $Y^{(1)}$ is simplicial (with simplicial $G$-action). Let $I^{\prime}$ be a set of representatives of the $G$-orbits of 0 -simplices, and let $I$ be the subset of $I^{\prime}$ consisting of those $i$ with $G_{i}$ nontrivial.

For $i$ in $I$, let $E(i)=\left\{e \in Y^{(1)} \backslash Y^{(0)} \mid i \in \bar{e}\right\}$ and $F(i)=\left\{\sigma \in Y \backslash Y^{(1)} \mid i \in\right.$ $\bar{\sigma}\}$. Note that $G_{i}$ freely permutes the cells of $E(i)$ [respectively, of $F(i)$ ]. Choose a set $F^{\prime}(i)$ of representatives of $F(i)$ modulo $G_{i}$. For $\sigma$ in $F^{\prime}(i)$, let $f_{\sigma}:\left(E^{2}, S^{1}\right) \rightarrow\left(Y, Y^{(1)}\right)$ be a characteristic map satisfying $f_{\sigma}(1)=i$. Thus $f_{\sigma}\left(S^{1}\right)$ is a closed path $e_{1} e_{2} \ldots e_{n}$ with $o\left(e_{1}\right)=i, t\left(e_{1}\right)=o\left(e_{2}\right), \ldots, t\left(e_{n-1}\right)=$ $o\left(e_{n}\right), t\left(e_{n}\right)=i$ where $o(e)$ [resp. $t(e)$ ] denotes the initial [resp. terminal] point of the edge $e$, and $n>2$ because $Y^{(1)}$ is simplicial.

Choose points $x_{\sigma}, y_{\sigma} \in S^{1}$ such that $f_{\sigma}\left(x_{\sigma}\right)=t\left(e_{1}\right)$ and $f_{\sigma}\left(y_{\sigma}\right)=o\left(e_{n}\right)$. Let $(x y)_{\sigma}$ denote the segment in $E^{2}$ that joins $x_{\sigma}$ with $y_{\sigma}$. Then $E^{2}=$ $E_{\sigma}^{+} \cup E_{\sigma}^{-}$, where $1 \in E_{\sigma}^{+}$, and $E_{\sigma}^{+} \cap E_{\sigma}^{-}=(x y)_{\sigma}$. We subdivide the 2-cell $\bar{\sigma}$ by introducing the 1-cell $f_{\sigma}\left[(x y)_{\sigma}\right]$ (with endpoints $t\left(e_{1}\right)$ and $o\left(e_{n}\right)$ ) and the two 2-cells $f_{\sigma}\left(\stackrel{\circ}{E}_{\sigma}^{+}\right)$and $f_{\sigma}\left(\stackrel{\circ}{E}_{\sigma}^{-}\right)$. Use the $G_{i}$-action to translate this subdivision to the cells $g \sigma, g$ in $G_{i}$, and repeat the same procedure for each $\sigma$ in $F^{\prime}(i)$. Doing this for each $i$ in $I$ gives $Y$ a new $C W$ structure, and the old $G$-action extends to a new cellular action of the same type as the old one. We continue to call it $Y$.

We can now define an analog of the star of $i$. Let $X_{i}$ be the smallest subcomplex of $Y$ containing $G_{i} \cdot\left\{f_{\sigma}\left(E_{\sigma}^{+}\right) \mid \sigma \in F^{\prime}(i)\right\}$. It follows from $H_{1}(Y, Y-i)=$ $\widetilde{H}_{0}\left(S^{1}\right)=0$ that $X_{i}$ contains $E(i) . X_{i}$ is a contractible (closed) neighborhood of $i$, whose boundary $\partial X_{i}$ is the graph with edges $G_{i} \cdot\left\{f_{\sigma}\left[(x y)_{\sigma}\right] \mid \sigma \in F^{\prime}(i)\right\}$. Both $X_{i}$ and $\partial X_{i}$ are $G_{i}$-subcomplexes of $Y$, with $G_{i}$ freely permuting every cell different from $i$. It is clear that $\partial X_{i}$ is a strong deformation retract of $X_{i}-i$, so that

$$
H_{*}\left(\partial X_{i}\right) \cong H_{*}\left(X_{i}-i\right) \cong H_{*+1}(Y, Y-i) \cong H_{*}\left(S^{1}\right),
$$

showing that $\partial X_{i}$ is a graph homotopy equivalent to $S^{1}$. It is then easy to find an injective simplicial homotopy equivalence $\psi: S^{1} \rightarrow \partial X_{i}$, and we set 
$\psi\left(S^{1}\right)=K_{i}$. It is plain that $\partial X_{i}$ (strong deformation) retracts to $K_{i}$, and that this retraction can be taken to be $G_{i}$-equivariant. Furthermore, we must have $G_{i} \cdot K_{i} \subset K_{i}$, for otherwise $H_{1}\left(\partial X_{i}\right)$ would be a free abelian group of rank $\geq 2$. Indeed, if $g e \notin K_{i}$ (for some $g$ in $G_{i}$ and some edge $e$ in $K_{i}$ ) then the subgroup $H_{1}\left(K_{i} \cup g K_{i}\right)$ of $H_{1}\left(\partial X_{i}\right)$ is free of rank at least 2.

Since $X_{i}$ and $C \partial X_{i}$ have isomorphic 1-skeletons, there is a map $X_{i}^{(1)} \rightarrow$ $C \partial X_{i}$ which can be extended (as in Lemma 7) to a $G_{i}$-equivariant map $X_{i} \rightarrow$ $C \partial X_{i}$. Also the $G_{i}$-equivariant retract $\partial X_{i} \rightarrow K_{i}$ extends, by coning, to a $G_{i}$-equivariant retract $C \partial X_{i} \rightarrow C K_{i}$, and we set $Z_{i}=C K_{i}$. Composition then gives a $G_{i}$-equivariant map $h_{i}: X_{i} \rightarrow Z_{i}$ which is a homotopy equivalence (because $X_{i}$ and $Z_{i}$ are contractible). We now let $Y^{\prime}$ denote the complex obtained from $Y$ by replacing the $X_{i}$ by the $Z_{i}$ ( $i$ in $I$ ). By Proposition $5, Y^{\prime}$ is a 2-dimensional contractible 0 -unfree $G$-complex. Notice that $G$ acts freely on $Y^{\prime}-Z$, where $Z=\bigsqcup_{I} G \times_{G_{i}} Z_{i}$. Thus, to obtain a complex as in (ii) from $Y^{\prime}$, we need only observe that each $Z_{i}$ is a 2-cell and that we may "forget" the cone structure, i.e., the subdivision of the interior of $Z_{i}$. The proof is complete.

Theorem 5 applies notably to fuchsian groups, as these are discrete groups of orientation-preserving isometries of the hyperbolic plane, which preserve some hyperbolic tesselation with the property that the only fixed points of the action are vertices of the tesselation.

\section{PAIRS OF COHOMOLOGICAL DIMENSION ONE}

This section is concerned with the case $n=1$ of Theorem 3. Recall that in this case the theorem can be stated as follows: $\left(G,\left(G_{i}\right)_{I}\right)$ has cohomological dimension one if and only if $G=*_{I} G_{i} * F$, for some free group $F$. The following Theorem of Dicks [Di, Theorem (2.11), p. 117] gives the special case when $I$ has only one element.

Theorem [Dicks]. $(G, H)$ has cohomological dimension one if and only if $G=$ $H * F$ for some free group $F$.

Theorem 3 (for $n=1$ ) will be deduced from Dicks' Theorem via a trick of Wall. For this we need a technical result, Proposition 8 below, which is inspired in [W 1, Theorem 2]. Our proof, however, is completely algebraic and valid for arbitrary $I$. This is done in (1) below. In (2) (certain) pairs of relative dimension one are characterized.

1. We recall some notation first. We assume given a pair $\left(G,\left(G_{i}\right)_{I}\right)$ from which we define $S$ and $\Delta$ in the usual way, denoting the inclusion $\Delta \rightarrow S$ by $j$. There are exact sequences (of $G_{i}$-modules) $0 \rightarrow I_{G_{i}} \rightarrow \mathbb{Z} G_{i} \rightarrow \mathbb{Z} \rightarrow 0$, where $I_{G_{i}}$ is the augmentation ideal. Applying $\mathbb{Z} G \otimes_{G_{i}}$ - we obtain the exact sequence $0 \rightarrow J_{i} \rightarrow \mathbb{Z} G \rightarrow \mathbb{Z} G / G_{i} \rightarrow 0$, where $J_{i}$ is short for $J_{G_{i}}(G)=\mathbb{Z} G \otimes_{G_{i}} I_{G_{i}}$. Taking 
the direct sum over $I$ yields the exact sequence of $G$-modules $0 \rightarrow \bigoplus_{I} J_{i} \stackrel{u}{\longrightarrow}$ $\mathbb{Z} G^{(I)} \stackrel{r}{\rightarrow} S \rightarrow 0$. There is also an augmentation $\varepsilon_{I}: \mathbb{Z} G^{(I)} \rightarrow \mathbb{Z}$ defined by $\varepsilon_{I}(g)=1$ for all $g$ in $G$. Denote its kernel by $\mathscr{J}$, and notice that there is an inclusion $k: \bigoplus_{I} J_{i} \rightarrow \mathcal{J}$. This is summarized in the following commutative diagram (with exact rows and right-hand column):

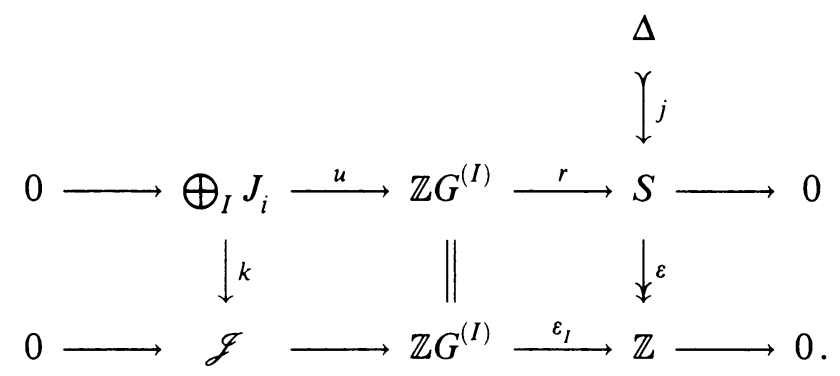

By the snake lemma, $0 \rightarrow \bigoplus_{I} J_{i} \stackrel{k}{\longrightarrow} \mathscr{J} \rightarrow \Delta \rightarrow 0$ (4) is exact too.

Suppose now that $I^{\prime}=I-\left\{i_{0}\right\}$ is not empty, and let $\bar{G}$ denote the free product of $G$ and infinite cyclic groups with generators $w_{i}, i$ in $I^{\prime}$. Let $H$ be the subgroup of $\bar{G}$ which is the free product of $G_{i_{0}}$ and the $G_{i}^{w_{i}}$ (for $\left.i \neq i_{0}\right)$. We write $H=*_{I} G_{i}^{w_{i}}$, where $w_{i_{0}}=1$. We have thus defined a new pair $(\bar{G}, H)$. The diagram corresponding to $(3)$ is

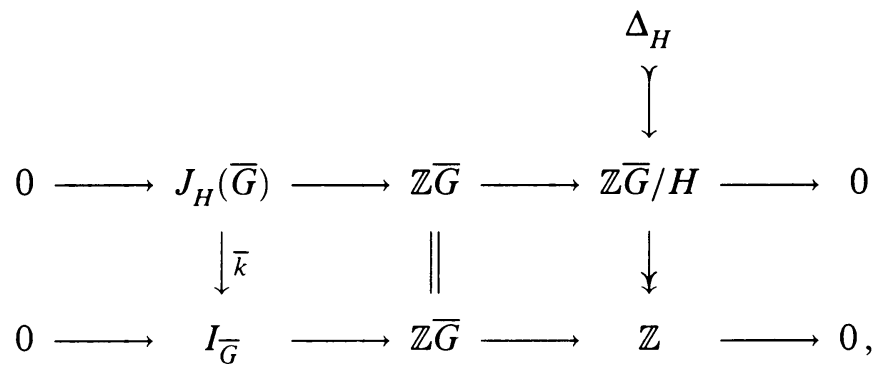

and $0 \rightarrow J_{H}(\bar{G}) \stackrel{\bar{k}}{\longrightarrow} I_{\bar{G}} \rightarrow \Delta_{H} \rightarrow 0$ (5) is exact.

Proposition 8. In the situation described above, we have

$$
\left.\Delta_{H} \cong \mathbb{Z} \bar{G} \otimes_{G} \Delta \quad \text { (as } \mathbb{Z} \bar{G} \text {-modules }\right) .
$$

Proof. We shall construct $\bar{G}$-module isomorphisms $\mu$ and $\mu^{\prime}$ so that

$$
\begin{aligned}
& 0 \longrightarrow \oplus J_{G_{i}}(\bar{G}) \stackrel{\mathrm{id} \otimes k}{\longrightarrow} \mathbb{Z} \bar{G} \otimes_{G} \mathcal{J} \longrightarrow \mathbb{Z} \bar{G} \otimes_{G} \Delta \longrightarrow 0 \\
& \simeq \mu^{\prime} \quad \simeq{ }^{\prime} \quad \simeq \mathfrak{\downarrow}^{\prime} \mu^{\prime \prime} \\
& 0 \longrightarrow J_{H}(\bar{G}) \stackrel{\text { incl. }}{\longrightarrow} I_{\bar{G}} \longrightarrow \Delta_{H} \longrightarrow 0
\end{aligned}
$$

commutes. The exact top row is (4) tensored with $\mathbb{Z} \bar{G} \otimes_{G}-$, and the bottom row is (5). It will thus follow that $\mu$ and $\mu^{\prime}$ induce the desired isomorphism $\mu^{\prime \prime}: \mathbb{Z} \bar{G} \otimes_{G} \Delta \rightarrow \Delta_{H}$. 
We begin by defining $\mu^{\prime}$. For each $i$ in $I$, define $f_{i}: \mathbb{Z} \bar{G} \rightarrow \mathbb{Z} \bar{G}$ by $f_{i}(g)=$ $g w_{i}^{-1}$, for $g$ in $\bar{G}$. Then $f_{i}$ is an isomorphism of (left) $\mathbb{Z} \bar{G}$-modules, with inverse given by $f_{i}^{-1}(g)=g w_{i}$. There are also maps $\bar{f}_{i}: \mathbb{Z} \bar{G} / G_{i} \rightarrow \mathbb{Z} \bar{G}_{i} / G_{i}^{w_{i}}$ given by $\bar{f}_{i}\left(g G_{i}\right)=g w_{i}^{-1} G_{i}^{w_{i}}$, which are $\mathbb{Z} \bar{G}$-isomorphisms and commute with the augmentations in the diagram below

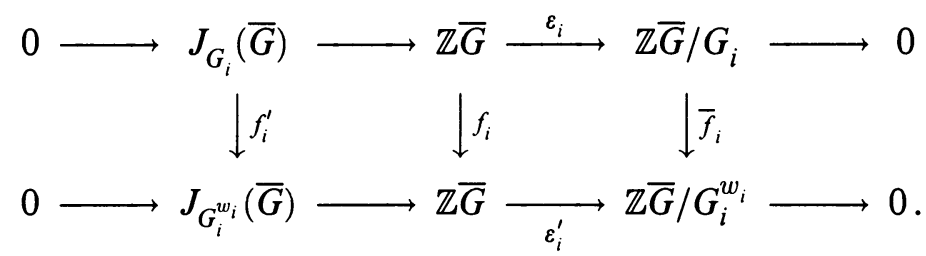

We thus obtain $\bar{G}$-isomorphisms $f_{i}^{\prime}$. Next, recall the augmentation ideal of a free product is the direct sum of the corresponding $J$ 's. More precisely, in the case of $H=*_{I} G_{i}^{w_{i}}$, the inclusions $J G_{i}^{w_{i}}(H) \rightarrow I_{H}$ induce an $H$-isomorphism $\bigoplus_{I} J G_{i}^{w_{i}}(H) \rightarrow I_{H}$. Tensoring this with $\mathbb{Z} \bar{G} \otimes_{H}-$ gives a $\bar{G}$-isomorphism $\alpha$ : $\bigoplus_{I} J G_{i}^{w_{i}}(\bar{G}) \cong J_{H}(\bar{G})$. We now define $\mu^{\prime}$ as the composite $\mu^{\prime}=\alpha\left(\bigoplus_{I} f_{i}^{\prime}\right)$.

To define $\mu$, begin with the short exact sequence

$$
0 \rightarrow I_{G} \oplus \mathbb{Z} G^{\left(I^{\prime}\right)} \rightarrow \mathbb{Z} G \oplus \mathbb{Z} G^{\left(I^{\prime}\right)} \stackrel{t}{\longrightarrow} \mathbb{Z} \rightarrow 0
$$

obtained from $0 \rightarrow I_{G} \rightarrow \mathbb{Z} G \rightarrow \mathbb{Z} \rightarrow 0$ by adding $0 \rightarrow \mathbb{Z} G^{\left(I^{\prime}\right)} \rightarrow \mathbb{Z} G^{\left(I^{\prime}\right)} \rightarrow 0$ to it. Define a map $\Phi: \mathbb{Z}^{(I)} \rightarrow \mathbb{Z} G \oplus \mathbb{Z} G^{\left(I^{\prime}\right)}$ by $\Phi\left(x_{i}\right)_{I}=\left(\sum_{I} x_{i},\left(-x_{i}\right)_{I^{\prime}}\right)$, where we have represented the elements of the direct sum $\mathbb{Z} G^{(I)}$ as $I$-tuples $\left(x_{i}\right)_{I}$. Note that $\sum x_{i}$ is a well-defined element of $\mathbb{Z} G$ because only finitely many $x_{i}$ are nonzero. $\Phi$ is a $\mathbb{Z} G$-homomorphism with inverse of the same form: $\Phi^{-1}\left(y_{i}\right)_{I}=\left(\sum_{I} y_{i},\left(-y_{i}\right)_{I^{\prime}}\right)$. It is clear that $t \Phi=\varepsilon_{I}$, so that there is a $\mathbb{Z} G$-isomorphism $\Phi^{\prime}: \mathcal{J} \rightarrow I_{G} \oplus \mathbb{Z} G^{\left(I^{\prime}\right)}$ (with the notation of (3)).

Next, let $W_{i}$ denote the infinite cyclic group generated by $w_{i}$, and recall that there is an exact sequence of $\mathbb{Z} W_{i}$-modules $0 \rightarrow \mathbb{Z} W_{i} \stackrel{\ell_{i}^{\prime}}{\longrightarrow} \mathbb{Z} W_{i} \stackrel{\varepsilon}{\longrightarrow} \mathbb{Z} \rightarrow 0$, where $\ell_{i}^{\prime}(1)=w_{i}-1$ (cf., e.g. [B, p. 16]). Thus $\ell_{i}^{\prime}: \mathbb{Z} W_{i} \cong I_{W_{i}}$ is an isomorphism, but then so is $\ell_{i}$, defined by $\ell_{i}(1)=1-w_{i}^{-1}$. The above short exact sequence yields, inducing to $\mathbb{Z} \bar{G}$, the exact sequence of $\mathbb{Z} \bar{G}$-modules $0 \rightarrow \mathbb{Z} \bar{G} \rightarrow \mathbb{Z} \bar{G} \rightarrow$ $\mathbb{Z} \bar{G} / W_{i} \rightarrow 0$, and the map corresponding to $\ell_{i}$ (which we continue to denote $\ell_{i}$ ) establishes a $\mathbb{Z} \bar{G}$-isomorphism $\ell_{i}: \mathbb{Z} \bar{G} \cong J_{W_{i}}(\bar{G})$. We now define $\mu$ as the composite of the following isomorphisms:

$$
\begin{aligned}
\mathbb{Z} \bar{G} \otimes_{G} \mathscr{J} \stackrel{\mathrm{id}_{\bar{G}}^{\otimes \Phi^{\prime}}}{\longrightarrow} \mathbb{Z} \bar{G} \otimes_{G}\left(I_{G} \oplus \mathbb{Z} G^{\left(I^{\prime}\right)}\right) \\
\quad=J_{G}(\bar{G}) \oplus \mathbb{Z} \bar{G}^{\left(I^{\prime}\right)} \stackrel{\text { id } \oplus\left(\oplus_{i}\right)}{\longrightarrow} J_{G}(\bar{G}) \oplus \bigoplus_{I^{\prime}} J_{W_{i}}(\bar{G}) \rightarrow I_{\bar{G}} .
\end{aligned}
$$

It remains only to check the commutativity of (6). If $i=i_{0}$ then all the maps involved are either inclusions or identities. If $i$ is in $I^{\prime}$, one checks easily that 
(6) commutes on the set $\left\{x g_{i}-x \mid x \in \bar{G}, g_{i} \in G_{i}\right\}$ of $\mathbb{Z}$-generators of $J_{G_{i}}(\bar{G})$. This proves commutativity and finishes the proof.

Corollary 9. Let $\left(G,\left(G_{i}\right)_{I}\right), \bar{G}$ and $H$ be as above, and $n$ a positive integer. Then $\operatorname{cd}\left(G,\left(G_{i}\right)_{I}\right) \leq n$ implies $\operatorname{cd}(\bar{G}, H) \leq n$.

Remark. The corollary for $n=1$ and finite $I$ is Theorem 2 of [W 1].

Proof of Theorem 3 for $n=1$. Let $\left(G,\left(G_{i}\right)_{I}\right)$ be a pair of cohomological dimension one. If $I$ has only one element we have exactly Dicks' Theorem. If $I$ has more than one element, apply the above construction to get the pair $(\bar{G}, H)$, which has $\mathrm{cd}=1$ by Corollary 9 . Then by Dicks' Theorem, $G=$ $H * F=*_{I} G_{i}^{w_{i}} * F$ for some free group $F$. The converse follows from Corollary 1. This completes the proof.

2. In this section we characterize certain groups of $\mathrm{rd}=1$.

Proposition 9. Let $G$ be a group such that the orders of its finite subgroups are bounded. Then the following conditions are equivalent:

(i) $\operatorname{rd} G \leq 1$.

(ii) $G \cong *_{I} G_{i} * F$ where $F$ is free and $\left(G_{i}\right)_{I}$ is a set of representatives of the conjugacy classes of maximal finite subgroups of $G$.

(iii) vcd $G \leq 1$ and $\mathrm{rd} G<\infty$.

Proof. The equivalence of (i) and (ii) is Theorem 3 for $n=1$, and holds with no extra assumptions on $G$. Let $G$ be as in (ii). Then vcd $G \leq 1$ by [S-W, Theorem 7.3] and $\mathrm{rd} G<\infty$ by Corollary 1. Finally, Proposition 2 shows that (iii) implies (i).

Example. Let $A$ and $B$ be finite groups of orders $a+1$ and $b+1$, respectively $(a, b>0)$. Then the kernel $K$ of the canonical homomorphism $A * B \rightarrow A \times B$ is the free group of rank $a b$ (see e.g. [S, Proposition 4]). Let $H=A * B * A *$ $B$, and $G=(A * B){ }_{K}(A * B)$. It follows from Corollaries 1 and 2 that rd $H=1$ and that $\operatorname{rd} G=\operatorname{cd}(G,(A, A, B, B)) \leq 2 . H$ and $G$ are not isomorphic because their Euler characteristics are different: $\chi(G)-\chi(H)=a b$. By Proposition 9, rd $G=2$. However, appropriate choices of $A$ and $B$ will produce groups $H$ and $G$ having equal qpd. For instance, taking $A=B=$ $\mathbb{Z} / 2 \mathbb{Z}$ (cf. [H-S 1, Example 4]) we still have $\operatorname{rd~} H=1$ and $\operatorname{rd} G=2$, but qpd $H=$ qpd $G=2$.

\section{REFERENCES}

[B-E] R. Bieri and B. Eckmann, Relative homology and Poincaré duality for group pairs, J. Pure Appl. Algebra 13 (1978), 277-319.

[B] K. S. Brown, Cohomology of groups, Graduate Texts in Math. 87, Springer, Berlin-Heidelberg-New York, 1982.

[C] I. M. Chiswell, Exact sequences associated with a graph of groups, J. Pure Appl. Algebra 8 (1976), 63-74. 
[Di] W. Dicks, Groups, trees and projective modules, Lecture Notes in Math., vol. 790, Springer, Berlin-Heidelberg-New York, 1980.

[Du] M. J. Dunwoody, Accessibility and groups of cohomological dimension one, Proc. London Math. Soc. (3) 38 (1979), 193-215.

[H-S 1] J. Howie and H. R. Schneebeli, Groups of finite quasi-projective dimension, Comment. Math. Helv. 54 (1979), 615-628.

[H-S 2] __ Cellular actions and groups of finite quasi-projective dimension, Math. Z. 170 (1980), 85-90.

[Hu] J. Huebschmann, Cohomology theory of aspherical groups and of small cancellation groups, J. Pure Appl. Algebra 14 (1979), 137-143.

[S] J.-P. Serre, Trees, Springer, Berlin-Heidelberg-New York, 1980.

[Sw 1] R. G. Swan, Periodic resolutions for finite groups, Ann. of Math. 72 (1960), 267-291.

[Sw 2] _ Groups of cohomological dimension one, J. Algebra 12 (1969), 585-601.

[S-W] P. Scott and C. T. C. Wall, Topological methods in group theory, London Math. Soc. Lecture Note Ser. 36 (1979).

[T] O. Talelli, On Cohomological periodicity for infinite groups, Comment. Math. Helv. 55 (1980), 178-192.

[W 1] C. T. C. Wall, Pairs of relative cohomological dimension one, J. Pure Appl. Algebra 1 (1971), 141-154.

[W 2] _ (Ed.), Homological group theory, London Math. Soc. Lecture Note Series 36, Cambridge University Press, Cambridge, 1979.

Department of Mathematics, University of Stockholm, P.O. Box 6701, S-113 85 StockHOLM, SWEDEN 